\title{
Solar migrating atmospheric tides in the winds of the polar region of Venus
}

\author{
J. Peralta $^{\mathrm{a}, *}$, D. Luz ${ }^{\mathrm{a}}$, D.L. Berry ${ }^{\mathrm{b}}$, C.C.C. Tsang ${ }^{\mathrm{c}}$, A. Sánchez-Lavega ${ }^{\mathrm{d}}$, R. Hueso ${ }^{\mathrm{d}}$, G. Piccioni $^{\mathrm{e}}$, P. Drossart $^{\mathrm{f}}$ \\ a CAAUL/Observatório Astronómico de Lisboa, Tapada da Ajuda, 1349-018 Lisboa, Portugal \\ ${ }^{\mathrm{b}}$ Departamento de Física, Universidade de Évora, 7002-554 Évora, Portugal \\ ${ }^{\mathrm{c}}$ Department of Space Studies, Southwest Research Institute, Boulder, CO 80302, USA \\ d Departamento de Física Aplicada I/Escuela Superior de Ingeniería, Universidad del País Vasco, Bilbao, Spain \\ e INAF/IAPS, 100 Via del Fosso del Cavaliere, Rome, Italy \\ ${ }^{\mathrm{f}}$ LESIA/Observatoire de Paris, CNRS UPMC, Univ. Paris-Diderot, 5, Place Jules Janssen, 92195 Meudon, France
}

\section{A R T I C L E I N F O}

\section{Article history:}

Received 27 January 2012

Revised 11 May 2012

Accepted 9 June 2012

Available online 4 July 2012

\section{Keywords:}

Atmospheres, Dynamics

Tides, Atmospheric

Venus

Venus, Atmosphere

\begin{abstract}
A B S T R A C T
We study the effects of migrating solar tides on the winds at the cloud tops of the polar region of Venus. The winds were measured using cloud tracking on images obtained at wavelengths of 3.9 and $5.0 \mu \mathrm{m}$ by the instrument VIRTIS-M onboard Venus Express. These wavelengths probe about the same altitude close to the cloud tops, allowing for the first time to retrieve winds simultaneously in the day and nightside of the planet. We use a dataset with observations from 16 orbits, covering a time span of 289 days and a latitude range between $70^{\circ} \mathrm{S}$ and $85^{\circ} \mathrm{S}$, the region where the so called cold collar resides. Diurnal and quarter-diurnal tides (wavenumbers 1 and 4 ) were detected in the wind field, with a decoupled influence on the zonal and meridional directions. The diurnal tide is the dominant harmonic with amplitudes of about $4.7 \mathrm{~m} / \mathrm{s}$ exclusively affecting the meridional component of the wind and forcing a solar-to-antisolar circulation at the polar region. The quarter-diurnal mode is only apparent in the zonal wind in a restricted latitude range with amplitudes $\sim 2.2 \mathrm{~m} / \mathrm{s}$. The spatial structure of the diurnal tide has also been investigated, obtaining a vertical wavelength of about $8 \mathrm{~km}$, in accordance with predictions by models. Finally, a theoretical relation between the amplitudes of tidal temperature and tidal wind has been derived and its validity tested with models and results from previous missions.
\end{abstract}

(c) 2012 Elsevier Inc. All rights reserved.

\section{Introduction}

The superrotation of the atmosphere of Venus is one of the most intriguing phenomena in planetary atmospheric dynamics, but after several decades and more than 25 missions to the planet, its mechanism still remains unclear. Soon after the rotation of Venus' atmosphere was discovered, Schubert and Whitehead (1969) suggested that superrotation could be produced by the slow motion of the subsolar point, a theory based on their laboratory results in which a moving bunsen flame heating a liquid could induce in it a mean flow. In the case of Venus, most of the sunlight absorption occurs between 45 and $70 \mathrm{~km}$ of altitude (Moroz et al., 1985), with the solar motion being eastwards and the atmosphere moving westwards. The waves thus excited will carry momentum away upwards and downwards from the region of excitation, consequently accelerating the fluid in the opposite direction the wave is moving, i.e. accelerating it in the retrograde sense (for a review see Gierasch et al., 1997). Following this idea, Fels and Lindzen (1974) showed that thermal excitation of internal gravity waves

\footnotetext{
* Corresponding author. Fax: +351 213616752

E-mail address: peralta@oal.ul.pt (J. Peralta).
}

could lead to mean accelerations of the fluid within the region of excitation.

The solar tides are global-scale gravity waves generated by the absorption of solar radiation (Holton et al., 2002). Such global oscillations have been observed in all types of atmospheric variables, including wind, temperature, pressure or density, and provide constraints upon the atmospheric structure (Lindzen and Chapman, 1969). In situ, remote sensing and ground-based observations have been used to confirm the presence of the solar tides in Venus (Forbes, 2004). First attempts came from Earth-based observations of the thermal infrared emission of the nightside (Ingersoll and Orton, 1974), observing a maximum in the brightness to the east of the antisolar point suggestive of tidal effects. Moreover, Apt et al. (1980) detected a strong solar-fixed component of the emission from 10.6 to $12.6 \mu \mathrm{m}$, corresponding to wavenumbers 1,2 (low altitudes) and 4 (high southern latitudes).

Data on the tides has later been inferred from infrared remote sensing of temperatures at the cloud tops and above. Main examples are the atmospheric temperatures inferred by the Pioneer Venus orbiter infrared radiometer (OIR) (Taylor et al., 1980; Schofield and Taylor, 1983), Venera-15 spectrometry (Zasova et al., 2002) and Venus Express' radio occultation instrument VeRa (Tellmann et al., 2009) and imaging spectrometer VIRTIS (Grassi et al., 
2010; Migliorini et al., 2012). The Pioneer Venus OIR data came from five filters covering the spectral range between 11.3 and $16.7 \mu \mathrm{m}$. It was found that wavenumbers 1 and 2 dominated (with wavenumber 3 seldom present) and wavenumber predominance was dependent on the latitude region. Venera- 15 confirmed the presence of the four tidal components (1-4) in the upper clouds and latitudes between $20^{\circ}$ and $70^{\circ}$ through both temperature and aerosol concentrations inferred from its measurements. These tidal harmonics were also detected and characterized both vertically and in latitude combining VIRA (Seiff et al., 1986) data with thermal results inferred by VEGA balloons, Magellan, Venera-15 and Venera-16 (Zasova et al., 2006, 2007). More recently, VeRa obtained temperature profiles for latitude ranges $75-85^{\circ}$ in both hemispheres, with the most prominent result being the wavenumber-2 tidal component at $\sim 62 \mathrm{~km}$ (Ignatiev et al., 2009). The solar tide has also been inferred from the cloud brightness distribution in dayside UV observations of the cloud tops (Del Genio and Rossow, 1990), suggesting that the changes in reflectivity could be related with vertical movements. A solar-fixed structure corresponding to wavenumber 1 was apparent in their results, with a brightness minimum and maximum found near the local noon at low and mid latitudes, respectively.

Wind measurements from cloud tracking in images taken during the Pioneer Venus mission (Rossow et al., 1990; Del Genio and Rossow, 1990; Limaye, 2007), the Galileo flyby (Belton et al., 1991; Toigo et al., 1994; Peralta et al., 2007) and also the Venus Express mission (Sánchez-Lavega et al., 2008; Moissl et al., 2009; Hueso et al., 2012) have also been used to characterize the solar tides. Zonal and meridional winds measured at low latitudes from the Pioneer Venus and Galileo observations are consistent with solar tides with wavenumbers 1 and 2, with the former having amplitudes comparable or stronger than the latter.

The vertical structure of the solar tides as well as their interaction with the mean circulation in Venus has been the subject of a number of simulation studies (Elson, 1983; Pechmann and Ingersoll, 1984; Fels, 1986; Shen and Zhang, 1990; Baker and Leovy, 1987; Takagi and Matsuda, 2005). These have allowed to estimate the vertical wavelengths of the main tidal components (wavenumbers 1 and 2), obtaining for the semidiurnal tide values of amplitude and phase consistent with OIR measurements (Pechmann and Ingersoll, 1984). Moreover, the tides have been shown to be a persistent feature in general circulation models of the Venus atmosphere (Hou et al., 1990; Newman and Leovy, 1992; Yamamoto and Takahashi, 2006; Takagi and Matsuda, 2007; Lebonnois et al., 2010).

In this paper we characterize the migrating solar tides from measurements of the wind field at the top of the clouds in Venus' southern polar region, a region that has been poorly covered by previous studies in terms of winds. To this purpose, we analyze the wind field obtained with automated cloud tracking in pairs of images taken by the VIRTIS-M instrument onboard Venus Express. In Section 2 we develop the equations and notation to be used throughout the paper. In Section 3, we describe the dataset used, introduce our study of the altitude sensitivities at the selected wavelengths, and describe the techniques followed to reduce the dispersion and carry out a spectral analysis of the measurements. In Section 4 we present a sensitivity analysis and characterize the tides and their effect on the winds and their latitudinal structure. In Section 5, the results are discussed and compared with previous studies, and the main conclusions are presented in Section 6 .

\section{Theoretical considerations}

The solar tides are Sun-synchronous global-scale gravity waves, with periods that are some integer fraction of the solar day (i.e. periods of $24,12,8,6$, etc., hours in local solar time). Since the aim of this work is to characterize the solar tides present in the wind field, which are expected to behave as periodic oscillations, a periodogram analysis followed by a fit to a trigonometric function was carried out; its mathematical notation is introduced below (Section 2.1). On the other hand, we also investigated the thermal structure of the solar tides by deriving a tidal equation between winds and temperature (Section 2.2).

\subsection{Notation for tidal fitting}

The general mathematical expression for an oscillation in a generic atmospheric parameter $F$ such as the wind velocity will be given by a series of harmonics, with each harmonic given by the following equation (Forbes, 2004):

$F^{m, j}(\lambda, \phi, z, t)=A^{m, j}(\phi, z) \cdot \cos \left(m \cdot \lambda-j \cdot \Omega \cdot t+\delta^{m, j}\right)$,

where $A^{m, j}$ is the amplitude for the disturbance in the corresponding atmospheric parameter, $j$ is a positive integer number to denote the different solar harmonics, $\Omega$ is the angular frequency of the phenomenon causing the tide (in this case, the apparent solar motion in Venus, i.e. 117 days), $t$ is the time, $\delta^{m j}$ is the tidal phase, $\lambda$ is the longitude, $\phi$ the latitude, and $m$ is the zonal wavenumber (i.e. the number of wave crests occurring along a latitude circle).

Since the solar tide is Sun-synchronous, it is seen by an observer on the ground as a wave propagating with the same speed as the apparent motion of the Sun and a phase velocity of $c_{p h}=-\Omega$. This implies that $c_{p h}=j \cdot \Omega / m$, then for solar tides $m=-j$ and Eq. (1) becomes:

$F^{j}(\lambda, \phi, z, t)=A^{j}(\phi, z) \cdot \cos \left(j \cdot[\Omega \cdot t+\lambda]+\delta^{j}\right)$,

Finally, in terms of local solar time $t_{L T}$, where $t_{L T}=t+\lambda / \Omega$ (Holton et al., 2002; Forbes, 2004), Eq. (2) becomes:

$F^{j}\left(\phi, z, t_{L T}\right)=A^{j}(\phi, z) \cdot \cos \left(j \cdot \Omega \cdot t_{L T}+\delta^{j}\right)$,

\subsection{Relation between tidal amplitudes in temperature and velocity}

In order to obtain a direct relation between the tidal amplitudes in temperature, pressure and wind velocity, we need to include the damping effect in the equations of motion. The processes responsible for the variability and damping of the solar tides are unclear: dynamical interactions with the background atmosphere or other planetary waves, or variations in the heating that forces the tides are possible candidates (Xu et al., 2009b). In fact, the diurnal tides can undergo amplifications by interacting with other gravity waves (Liu et al., 2008), or tidal damping due to turbulent viscosity from wave breaking, to nonlinear interactions with planetary waves or even with other tidal harmonics. These interactions are usually characterized by a Rayleigh friction coefficient $\left(K_{R}\right)$, with the amplification or damping occurring when this coefficient becomes negative or positive (Xu et al., 2009b).

$\mathrm{Xu}$ et al. (2009a, 2009b) derived a general equation for the solar migrating tide, under the assumptions that the mean meridional and vertical wind components are negligible $(\bar{v} \approx 0, \bar{w} \approx 0)$ and adding forcing terms of the form $X^{\prime}=-K_{R} \cdot u^{\prime}$ and $Y^{\prime}=-K_{R} \cdot v^{\prime}$ (where $u^{\prime}$ and $v^{\prime}$ are the zonal and meridional disturbances). In the case of the specific atmospheric region in our study, we introduce additional assumptions in order to obtain a direct relation between the tidal velocity and temperature disturbances for the diurnal tides. The horizontal and meridional momentum equations for the tides in spherical coordinates, neglecting the Coriolis terms and the products between velocity disturbances, are (Holton, 2004): 
$\left(\frac{\partial}{\partial t}+\frac{\bar{u}}{a \cdot \cos \phi} \frac{\partial}{\partial \lambda}\right) \cdot u^{\prime}+\left(\frac{1}{a} \frac{\partial \bar{u}}{\partial \phi}-\frac{\bar{u}}{a} \tan \phi\right) \cdot v^{\prime}+\left(\frac{\partial \bar{u}}{\partial z}+\frac{\bar{u}}{a}\right)$.

$w^{\prime}=-\frac{1}{\rho_{0} \cdot a} \cdot \frac{1}{\cos \phi} \frac{\partial p^{\prime}}{\partial \lambda}-K_{R} \cdot u^{\prime}$,

$\left(\frac{2 \cdot \bar{u}}{a} \tan \phi\right) \cdot u^{\prime}+\left(\frac{\partial}{\partial t}+\frac{\bar{u}}{a \cdot \cos \phi} \frac{\partial}{\partial \lambda}\right) \cdot v^{\prime}=-\frac{1}{\rho_{0} \cdot a} \cdot \frac{\partial p^{\prime}}{\partial \phi}-K_{R} \cdot v^{\prime}$

where $\rho_{0}$ is the atmospheric density, $a$ is the radius of Venus, $\bar{u}$ is the mean zonal flow, $\phi$ is the latitude, $K_{R}$ is the Rayleigh friction coefficient, $p^{\prime}$ is the pressure disturbance due to the tide, and $u^{\prime}$, $v^{\prime}, w^{\prime}$ are the zonal, meridional and vertical tidal disturbances, respectively. The physical parameters affected by the migrating tides adopt the general form of Eq. (2), which can also be expressed in terms of a complex exponential:

$F^{j}(\lambda, \phi, z, t)=A^{j}(\phi, z) \cdot e^{i \cdot\left[(\Omega \cdot t+\lambda) \cdot j+\delta^{j}\right]}$,

where $j=1,2,3, \ldots$ is the index for the diurnal, semidiurnal, terdiurnal, etc., solar harmonics, $\Omega$ is the angular frequency for the Venus solar day and $A^{j}(\phi, z)$ is the amplitude. Focusing on the diurnal tide $(j=1)$, we will have the following expressions for the tidal disturbances:

$u^{\prime}(\lambda, \phi, z, t)=U^{\prime}(\phi, z) \cdot e^{i \cdot(\Omega \cdot t+\lambda+\delta)}$,

$v^{\prime}(\lambda, \phi, z, t)=V^{\prime}(\phi, z) \cdot e^{i \cdot(\Omega \cdot t+\lambda+\delta)}$,

$W^{\prime}(\lambda, \phi, z, t)=W^{\prime}(\phi, z) \cdot e^{i \cdot(\Omega \cdot t+\lambda+\delta)}$,

$p^{\prime}(\lambda, \phi, z, t)=P^{\prime}(\phi, z) \cdot e^{i \cdot(\Omega \cdot t+\lambda+\delta)}$,

Replacing the disturbances Eqs. (6a-d) in Eqs. (4a) and (4b) and keeping only the amplitudes of the tidal disturbances, the following horizontal and meridional momentum equations are obtained:

$$
\begin{aligned}
& {\left[i \cdot\left(\Omega+\frac{\bar{u}}{a \cdot \cos \phi}\right)+K_{R}\right] \cdot U^{\prime}+\left(\frac{1}{a} \frac{\partial \bar{u}}{\partial \phi}-\frac{\bar{u}}{a} \tan \phi\right) \cdot V^{\prime}} \\
& \quad+\left(\frac{\partial \bar{u}}{\partial z}+\frac{\bar{u}}{a}\right) \cdot W^{\prime}=-\frac{i}{\rho_{0} \cdot a} \cdot \frac{P^{\prime}}{\cos \phi}
\end{aligned}
$$

$\left(\frac{2 \cdot \bar{u}}{a} \tan \phi\right) \cdot U^{\prime}+\left[i \cdot\left(\Omega+\frac{\bar{u}}{a \cdot \cos \phi}\right)+K_{R}\right] \cdot V^{\prime}=-\frac{1}{\rho_{0} \cdot a} \cdot \frac{\partial P^{\prime}}{\partial \phi}$,

Combining Eqs. (7a) and (7b), we obtain the following equations for the real and imaginary components:

$$
\begin{aligned}
& {\left[\left(\frac{2 \cdot \bar{u}}{a} \tan \phi\right)+K_{R}\right] \cdot U^{\prime}+\left(\frac{1}{a} \frac{\partial \bar{u}}{\partial \phi}-\frac{\bar{u}}{a} \tan \phi+K_{R}\right) \cdot V^{\prime}} \\
& \quad+\left(\frac{\partial \bar{u}}{\partial z}+\frac{\bar{u}}{a}\right) \cdot W^{\prime}=-\frac{1}{\rho_{0} \cdot a} \cdot \frac{\partial P^{\prime}}{\partial \phi}
\end{aligned}
$$

$\left(\Omega+\frac{\bar{u}}{a \cdot \cos \phi}\right) \cdot\left(U^{\prime}+V^{\prime}\right)=-\frac{1}{\rho_{0} \cdot a} \cdot \frac{P^{\prime}}{\cos \phi}$.

Using the equation of state for ideal gases $P_{0}=\rho_{0} R T_{0}$, Eq. (8b) can be rewritten as:

$\frac{P^{\prime}}{P_{0}}=-\frac{1}{R \cdot T_{0}} \cdot(\bar{u}+\Omega \cdot a \cdot \cos \phi) \cdot\left(U^{\prime}+V^{\prime}\right)$.

Assuming that the VIRTIS-M infrared images at the chosen wavelengths provide information of the thermal emission from atmospheric surfaces of constant density, or isosteres (Houghton,
2002), the amplitude of the thermal disturbance will be a function of the pressure disturbance only: $P^{\prime}=\rho_{0} R T^{\prime}$; applying the equation of state for ideal gases again to (9) we obtain:

$T^{\prime}=-\frac{1}{R} \cdot(\bar{u}+\Omega \cdot a \cdot \cos \phi) \cdot\left(U^{\prime}+V^{\prime}\right)$.

Thus, the amplitude for the temperature tidal disturbance can be computed from the amplitudes for wind velocity disturbances and from the mean zonal flow, which implies that the tidal thermal structure can be fully derived using the wind velocities alone. The validity for this expression is studied in Section 5.4.

\section{Dataset and analysis}

\subsection{Dataset description}

For the detection and characterization of solar tides at the altitude of the cloud tops of Venus, we used a dataset complementary of the one used by Luz et al. (2011). The winds were determined using the automated cloud-tracking technique previously validated in Luz et al. (2008), with the cross-correlation algorithm described in the "Supporting Online Material" by Luz et al. (2011). The algorithm was applied to pairs of images taken by the VIRTIS-M imaging spectrometer onboard Venus Express (Drossart et al., 2007; Svedhem et al., 2007). Wavelengths of 3.9 and $5.0 \mu \mathrm{m}$ were selected for this study as they allow the detection of the solar tides using cloud top winds from the day and nightside simultaneously, and thus permitting the detection of the diurnal tide.

The dataset of VIRTIS-M nadir images included observations from 16 orbits, covering a time span of 289 days in the latitude range $70-85^{\circ} \mathrm{S}$. As a consequence of using an automatic technique for cloud tracking, a small number of poor quality wind measurements may be present in the dataset and could increase its dispersion. As the number of outliers in the cloud-tracked windspeeds are a minor fraction of the total (the quality of the measurements is similar to the one in Luz et al. (2011)), we decided to filter the dataset using a weighted mean instead of the generally used arithmetic mean (this technique is frequently used also for the terrestrial atmosphere, see Holton et al., 2002). For every latitude interval the mean of the velocity is:

$\bar{u}=\sum_{i} w_{i} \cdot u_{i}$

where the weights $w_{i}$ associated to each particular measurement $u_{i}$ are not equal but proportional to the latitudinal density of measurements.

After computing the weighted mean, velocities differing from this mean by more than twice the corresponding standard deviation were considered as outliers and discarded. The resulting wind sample is, then, used to calculate a new weighted mean and the procedure is repeated until values higher than twice the standard deviation are no longer found. In all latitudes we only needed two steps to satisfy this condition, and this filter has only been applied to the zonal component of the wind, keeping the associated meridional component for the analysis. A summary of the resulting dataset is presented in Table 1, while the number of wind measurements distributed in latitude and local time is shown in Fig. 1A, and the zonally averaged zonal and meridional wind speeds in Fig. 1B.

Table 1 shows that a total of 14,341 windspeeds were available after the filtering step. In most of the orbits the local time sampling is sufficient to cover $24 \mathrm{~h}$ of local time, though the local time resolution is variable (see Fig. 1A). The right panel shows the meridional profiles for zonally averaged zonal (black triangles) and meridional winds (grey circles). The error bars represent the standard deviation and vary between 10 and $20 \mathrm{~m} / \mathrm{s}$, depending on the latitude. 
Table 1

Summary of wind measurements after the filtering process. The dataset of VIRTIS-M nadir images used in this work include observations from 16 orbits covering a time span of 289 days, and a total of 14,341 wind vectors. Within each orbit, several pairs of images were used in most of the cases and when possible. The coverage in latitude and local time is also indicated. While most of the wind measurements were obtained from pairs of images taken at $3.9 \mu \mathrm{m}$, data from images at $5.0 \mu \mathrm{m}$ were used in orbits 475 and 478

\begin{tabular}{llcll}
\hline $\begin{array}{l}\text { VEX } \\
\text { orbit }\end{array}$ & $\begin{array}{l}\text { Wavelength } \\
(\mu \mathrm{m})\end{array}$ & $\begin{array}{l}\text { Wind } \\
\text { measurements }\end{array}$ & $\begin{array}{l}\text { Latitude } \\
\text { coverage }\left({ }^{\circ} \mathrm{S}\right)\end{array}$ & $\begin{array}{l}\text { Local time } \\
\text { coverage }(\mathrm{h})\end{array}$ \\
\hline 355 & 3.9 & 971 & $70-85$ & $15-08$ \\
388 & 3.9 & 895 & $70-85$ & $00-24$ \\
390 & 3.9 & 747 & $70-85$ & $00-24$ \\
392 & 3.9 & 630 & $70-85$ & $00-24$ \\
394 & 3.9 & 794 & $70-85$ & $00-24$ \\
396 & 3.9 & 758 & $70-84$ & $00-24$ \\
398 & 3.9 & 671 & $70-85$ & $00-24$ \\
474 & 3.9 & 1114 & $70-85$ & $00-24$ \\
475 & 5.0 & 1093 & $70-85$ & $00-24$ \\
477 & 3.9 & 1009 & $70-85$ & $00-24$ \\
478 & 5.0 & 1909 & $70-85$ & $00-24$ \\
479 & 3.9 & 916 & $70-85$ & $00-24$ \\
638 & 3.9 & 649 & $70-85$ & $00-24$ \\
640 & 3.9 & 766 & $70-85$ & $00-24$ \\
642 & 3.9 & 793 & $70-85$ & $00-24$ \\
644 & 3.9 & 626 & $70-85$ & $00-24$ \\
\hline
\end{tabular}

\subsection{Altitude sensitivities}

When tracking cloud features on Venus, either in the near-infrared (at 1.74 and $2.3 \mu \mathrm{m}$ ) or at thermal infrared wavelengths (at 3.9 and $5.0 \mu \mathrm{m}$ ), it is important to know where the radiation originates from. This allows us to relate the derived wind vectors from feature tracking with an associated altitude of the flow. The radiative transfer model described in Tsang et al. (2008) has been used to calculate the altitude from which the thermal radiation originates in the 3.9 and $5.0 \mu \mathrm{m}$ regions. Fig. 2 displays the results of these calculations for both dayside and nightside observations, resulting in a minimal differential altitude when sounding with these two wavelengths at both day and nightside. These contribution functions are calculated for mode 1 sulfuric acid haze particles, with mean effective radius of $0.3 \mu \mathrm{m}$ and variance of $0.44 \mu \mathrm{m}$. The vertical profile for our cloud particles is derived from Pollack et al.
(1993) and demonstrates that the most abundant particle at the cloud top region is the mode 1 hazes (hence our calculation of the contribution function for this species).

The lower panel shows that the pure nightside thermal emission originating from the cloud tops at 3.9 and $5.0 \mu \mathrm{m}$ comes from the same altitude of approximately $60 \mathrm{~km}$, although for $5.0 \mu \mathrm{m}$ the weighting function is slightly broader in altitude than at $3.9 \mu \mathrm{m}$. The upper panel shows the dayside thermal emission. At $5.0 \mu \mathrm{m}$ the contribution function does not change much with height compared to the pure nightside case, but at $3.9 \mu \mathrm{m}$ an additional scattering layer is present at the cloud tops, thus displaying a double lobe contribution produced by both the thermal radiation from the cloud itself (lower lobe) and the scattering of solar radiation (upper lobe). Indeed, as we decrease in wavelength down to $3 \mu \mathrm{m}$, the scattering of solar photons becomes more prominent, as it is expected for shorter wavelengths (for which the smaller particles are more sensitive). This double lobe for the dayside at $3.9 \mu \mathrm{m}$ produces part of the dispersion observed in our results, although it we tested that the cloud features used for the tracking belong to the lower lobe and are the same as the observed at $5 \mu \mathrm{m}$ in dayside.

\subsection{Spectral analysis of the winds}

Our first objective has been to obtain samples of velocity disturbances in solar-fixed coordinates at different intervals of latitude. This is done in two steps: first, we divide the latitude region in intervals of $2^{\circ}$ (to facilitate the comparison with zonally averaged wind from other works) and we calculate the zonal mean of the zonal and meridional wind components (see Fig. 1B); then, we extract the wind disturbances by subtracting the zonal mean from the instantaneous flow (i.e. the zonal disturbances will be the wind departure from the zonal mean), $u^{\prime}=u-\bar{u}$, and the same applies for the meridional disturbances. Second, the velocity disturbances are sorted along a grid of local time intervals of $0.5 \mathrm{~h}$ and a new average is done for each local time interval. The result at each latitude interval and solar-fixed coordinates is the disturbances in the zonal and meridional components of the wind.

The spectral analysis of each solar-fixed series of wind velocity disturbances was carried out by computing a Lomb-Scargle periodogram (Scargle, 1982; Press and Rybicki, 1989). This periodo-
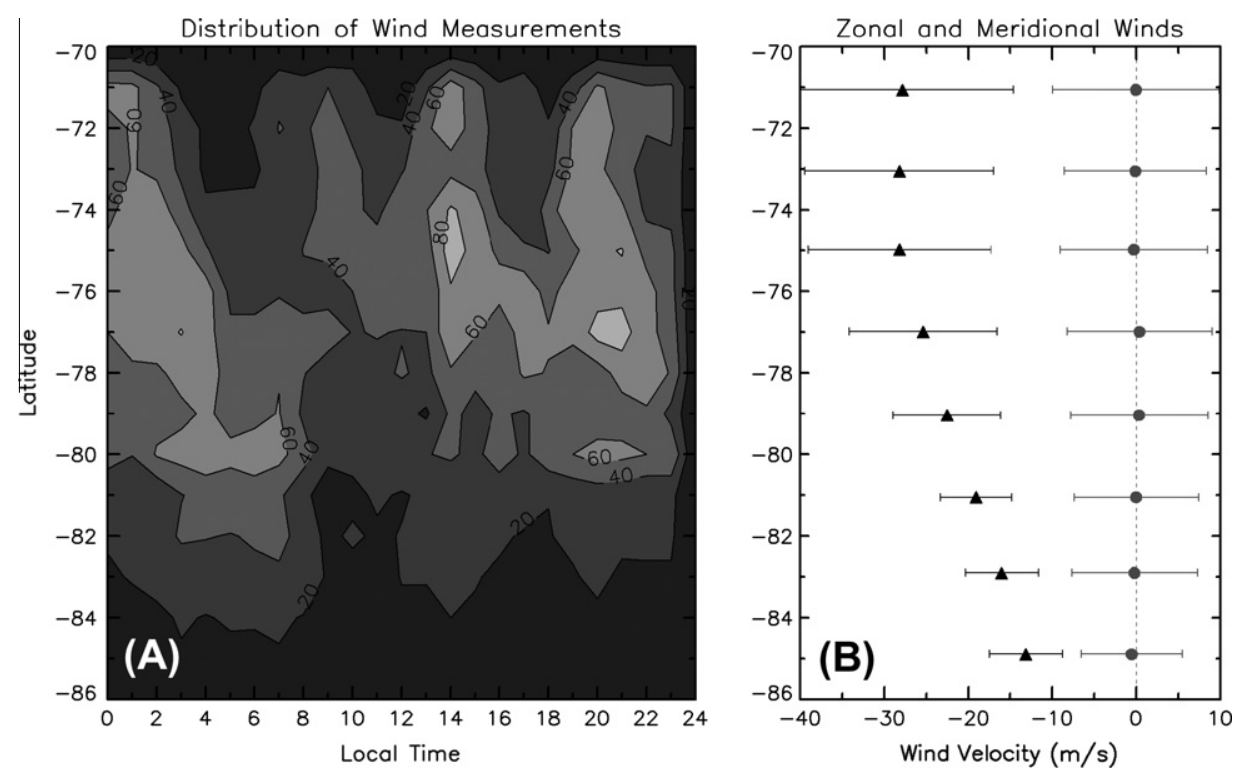

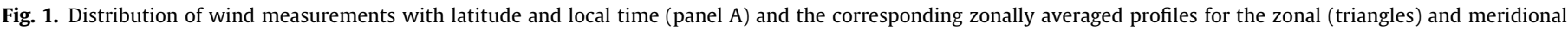
(circles) components of the wind (panel B). Both panels show post-filtering measurements. 

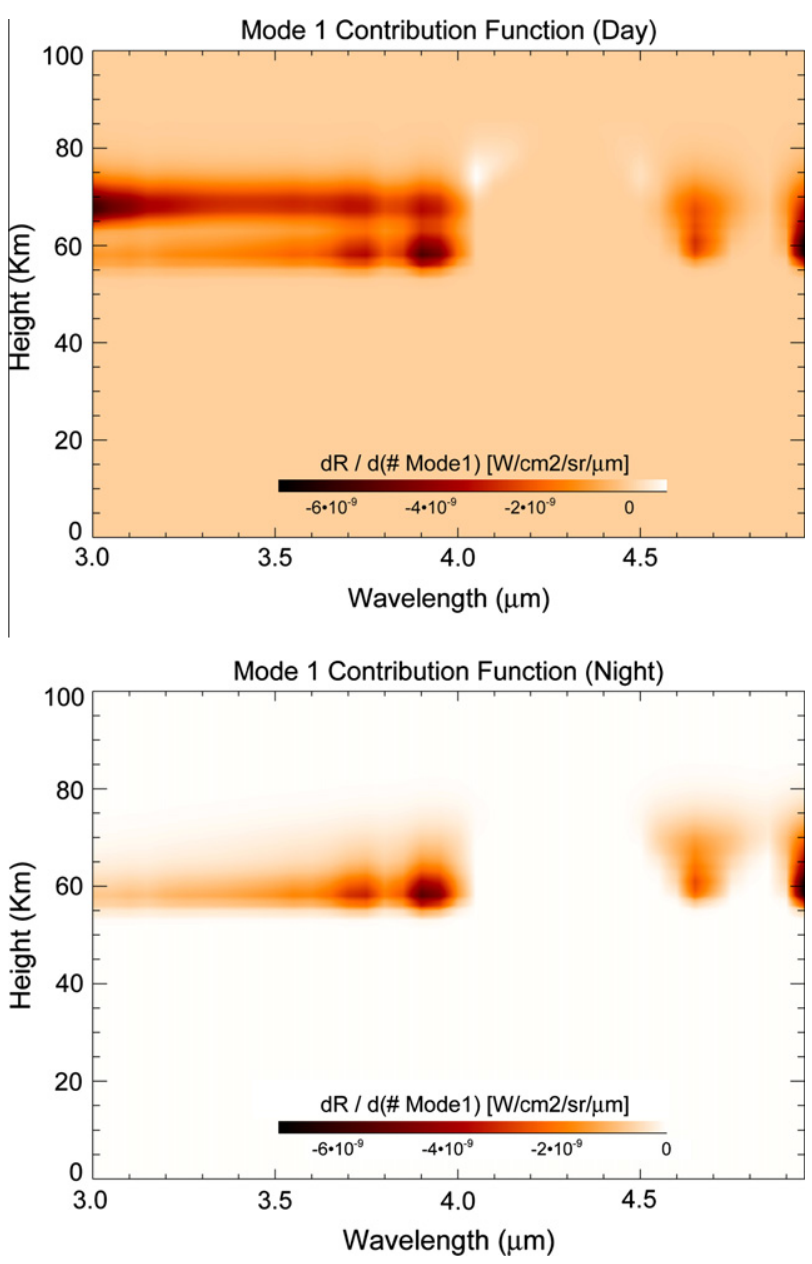

Fig. 2. The Venus mode1 (haze) contribution function from 3.0 to $5.0 \mu \mathrm{m}$, with the dayside in the upper panel and nightside in the lower one. Units are described as the amount of radiance change per unit change of particle density. The nightside emissions from 3.9 and $5.0 \mu \mathrm{m}$ probe the same altitude, centred at about $60 \mathrm{~km}$. The lack of emission between 4.0 and $4.5 \mu \mathrm{m}$ is due to strong $\mathrm{CO}_{2}$ absorption at these wavelengths. For the dayside thermal emission, the contribution function at $5.0 \mu \mathrm{m}$ is similar to the nightside emission, but the $3.9 \mu \mathrm{m}$ emission is distinctly different, owing to the more effective scattering of small haze particles at the cloud tops at shorter wavelengths.

gram estimates the spectral density of a signal, and when prominent peaks are present their corresponding frequencies are usually associated with the presence of periodic signals. In order to discard false candidates due to noise, the algorithm uses white noise simulations to calculate the power associated with a set of values of False Alarm Probability (hereafter FAP), i.e. the probability that a signal is exclusively due to noise (Frescura et al., 2007). In our periodograms, we only considered peaks with power values higher than a confidence level of $50 \%$ (which corresponds to a FAP of $1 \%$ ).

However, power values above the confidence level may not be associated with the presence of real periodic signals, as the FAPs only provide the probability that the signal is not caused by pure noise (Frescura et al., 2007). For this reason, if a candidate peak was detected, we confirmed the presence of a real periodic signal by fitting it to a generic sine function using the LevenbergMarquardt algorithm (Press et al., 1992). For the sine function, the integer $j$ is considered a fixed parameter with the value of the solar tide harmonic closest to the significant peak in the periodogram (see Eq. (3)). The fit was carried out by varying three main parameters: the amplitude, the phase and the offset relative to the mean flow.

\subsection{Inference of the horizontal and vertical tidal structure}

Once the solar tides are characterized through the zonal and meridional components of the wind at different latitudes, an estimation of both their horizontal and vertical structure was also carried out. With respect to the horizontal structure, we took advantage of the fact that we were dealing with global-scale waves and we fitted the tidal amplitudes with a series of normalized Legendre polynomials in a similar way as it is carried out for atmospheric parameters on the Earth (Lindzen and Chapman, 1969). As to the vertical structure, only the vertical wavelength was estimated using the dispersion equation from the Taylor-Goldstein equation in the case of long gravity waves in a fluid with constant Brunt-Väisälä frequency $N$ and a constant background wind (Nappo, 2002):

$$
k_{z}=\frac{N}{\left|j \cdot \Omega-u_{0} k_{x}\right|} \sqrt{k_{x}^{2}+k_{y}^{2}}
$$

where $j$ is the index for the solar harmonics and $k_{x}, k_{y}, k_{z}$ are the zonal, meridional and vertical wavenumbers respectively. It is important to mention that in the case of the Earth, Lindzen (1966) showed that many of the physical parameters affected by the solar tides are better described at all latitudes when fitted to an expansion of Hough functions instead of Legendre polynomials. We tested the applicability of the Hough functions to describe the meridional structure of the tidal disturbances on wind velocity and temperature, but found no realistic set of Hough functions that described well our results. This makes sense as in Venus the mean zonal wind is not small compared to the zonal phase speed of the tide (Lindzen, 1970), invalidating one of the most important assumptions in deriving the Laplace Tidal Equation (LTE) (see Appendix A for a review of the classical LTE). Since it is not the aim of this work to carry out a more precise development of a general tidal theory for Venus (Fels, 1986) but to obtain a gross estimation of the tidal structure, we restricted our tidal horizontal structure to the simpler description in terms of Legendre polynomials, as is shown in the next section. According to this classical formalism, an atmospheric parameter could then be described over a sphere, at an approximate vertical level, as:

$F(\cos \theta) \approx \sum_{n} C_{n} \cdot P_{s}^{n}(\cos \theta)$,

where $\theta$ is the co-latitude, $P_{s}^{n}$ is the Legendre polynomial for a zonal wavenumber $s$ and a meridional wavenumber $(|n|-s)$, and $C_{n}$ is the Legendre coefficient for the index $n$.

\section{Results}

As a first approach to the study of the harmonic behaviour in the solar-fixed wind disturbances we computed their corresponding confidence maps. These were obtained by dividing the available latitude range in intervals of $1^{\circ}$ (the shortest possible interval due to our sample of wind measurements). Then, we obtained the solar-fixed wind disturbances within the latitude bins, computed the Lomb-Scargle periodogram, and determined the confidence level corresponding to each power value. Fig. 3 shows the confidence maps for the zonal and the meridional components of the wind.

The contour maps indicate the regions of latitude and wavenumber where we find power values above distinct confidence levels. The confidence maps for both components of the wind exhibit important differences, with the meridional one having higher levels of confidence. In the case of the zonal component, peaks are present in restricted regions of latitude with power values higher 

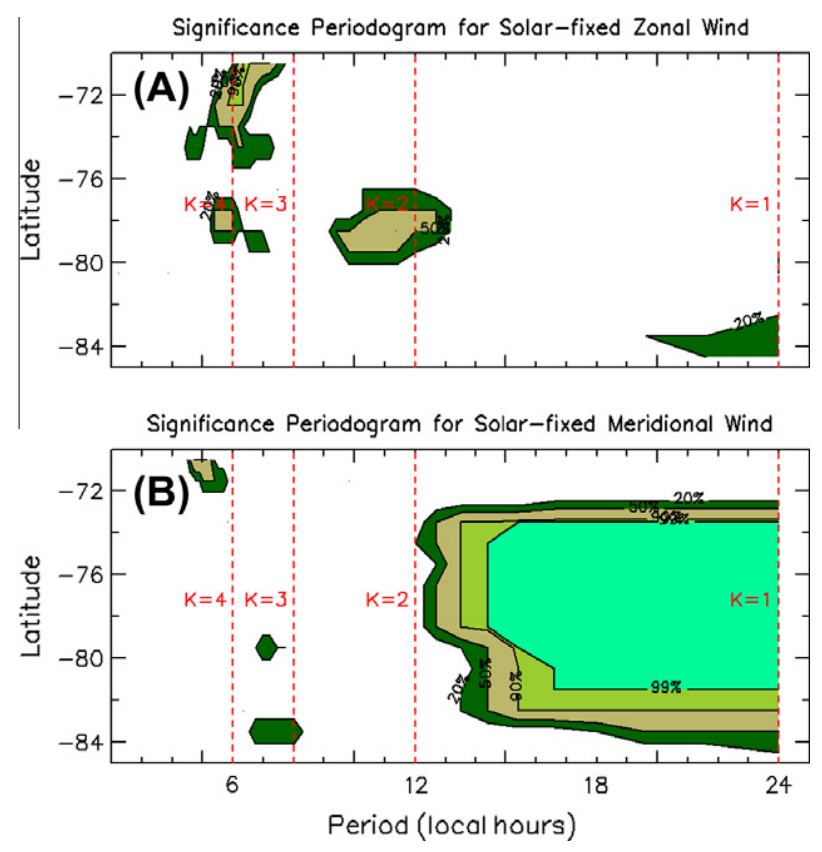

Fig. 3. Significance maps for Lomb-Scargle periodograms inferred from zonal (panel A) and meridional (panel B) solar-fixed wind disturbances in the latitude interval $70-85^{\circ} \mathrm{S}$. The data were averaged in latitude bins of $1^{\circ}$. The main solar tide harmonics are marked with dashed vertical lines.

than $50 \%$ and $90 \%$ of confidence for the semidiurnal and quarterdiurnal tides (periods of 12 and $6 \mathrm{~h}$ ); for the meridional wind the diurnal harmonic can be clearly detected with good confidence in a wide region of latitudes. The width of the most significant peaks can be explained as a result of the sampling in local time, as will be shown in Section 4.1. The decay in significance for the lower and higher values of latitude was expected as a consequence of the higher dispersion of the wind measurements in those regions.

\subsection{Sensitivity tests for periodograms}

In order to interpret the periodograms a sensitivity study was made of the effect of sampling resolution and noise on a pure sine signal. Examples analogous to tidal cases with the diurnal $(n=1)$ and semidiurnal $(n=2)$ periodicities are examined in Fig. 4.

The effect of the sampling resolution is analyzed in panel $4 \mathrm{~A}$, while panel $4 \mathrm{~B}$ shows the dependence on different levels of random noise. Both display the Lomb-Scargle periodograms for synthetic sinusoidal functions with wavenumbers 1 and 2 . No changes are apparent in peak positions or widths at half-maximum (around $7 \mathrm{~h}$ ). As the peak's maximum clearly increases with sampling resolution while the width at half-maximum is nearly constant, the sampling resolution seems only to affect to the peaks' kurtosis. Sampling resolution also affects the skewness, with worse resolution increasing the skewness to positive values and enlarging the right wing of the peak curve.

The effect of noise in the periodograms is analyzed in panel 4B. In this case, a sampling resolution of $0.5 \mathrm{~h}$ was chosen. The noise level is defined as a percentage of the amplitude of the synthetic sine function. It clearly has a higher impact than the sampling in local time. A higher noise level changes the peak's width at halfmaximum, increasing the value up to $2 \mathrm{~h}$. As in the previous case, no changes are apparent in peak positions.

\subsection{Characterization of the tidal effect on the winds}

As explained previously, in order to confirm the presence of periodic behaviour and to characterize it, a fit to a generic sine
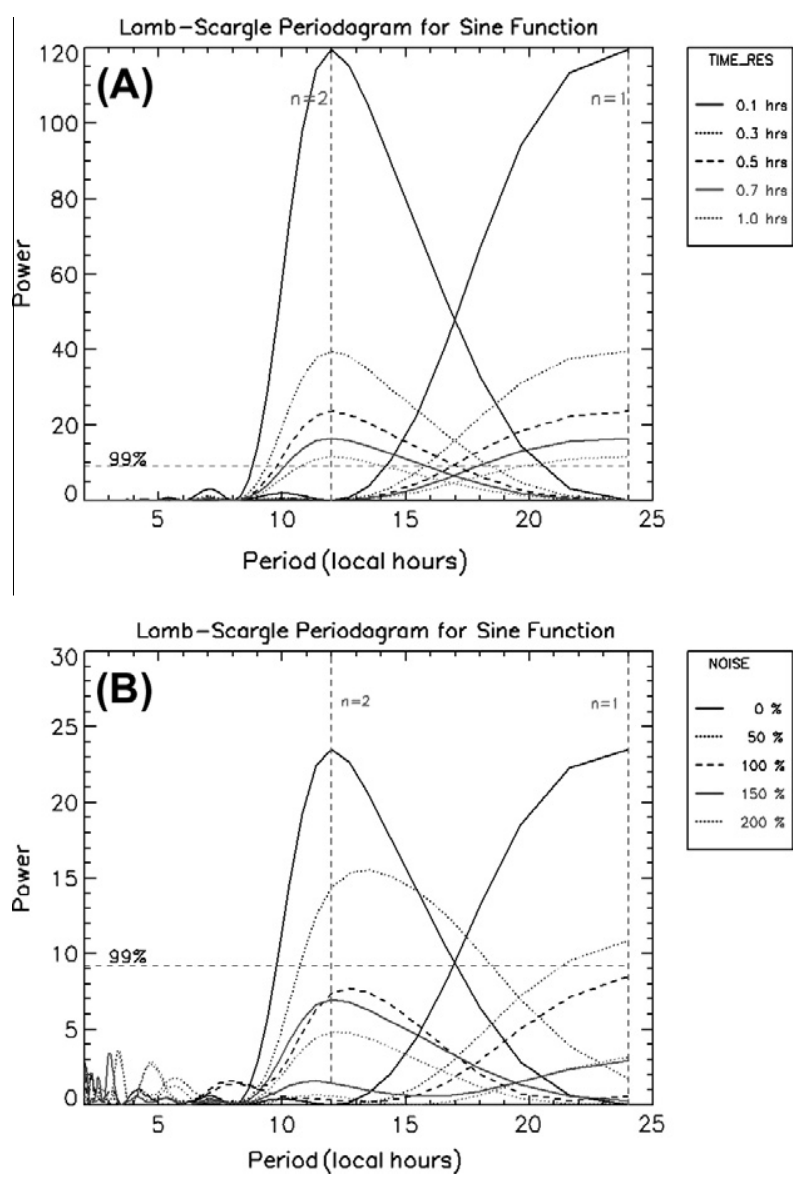

Fig. 4. Lomb-Scargle periodograms for synthetic sinusoidal functions with wavenumbers 1 and 2, for different sampling/local-time resolutions (panel A) and added noise (panel B). The confidence level of $99 \%$ is also marked. Harmonics 1 and 2 are marked with dashed vertical lines.

function was carried out (Eq. (3)), based on the results from the periodograms. As the quality and number of wind measurements changed with both latitude and orbit number, the search for solar tides was carried out using all the orbits together, which provided an increased signal to noise ratio. In Venus, we expect that the presence of the solar tides harmonics will not be affected by seasonal variations even over a time span of 289 days, since Venus lacks the main sources of tidal changes that affect the Earth (varying rate of orbital motion and inclination of rotation axis).

A full analysis of the zonal tidal disturbances has been carried out but results are conclusive only in a limited region of latitude where the periodograms indicate the presence of a quarter-diurnal harmonic in the zonal wind structure (power above the $95 \%$ confidence level). An example of this analysis is shown in Fig. 5 for the latitude interval $72-73^{\circ} \mathrm{S}$ and exhibiting a tidal amplitude of $\sim 5.0 \mathrm{~m} / \mathrm{s}$, what is up to a $20 \%$ of the zonally averaged zonal flow (see Fig. 1B).

The tidal effects on the meridional component of the wind are much stronger and with a higher power level. Fig. 6 displays the periodogram analyses and corresponding sine-fits. The wavenumber 1 mode (diurnal tide) is clearly detected and dominates this component of the wind. The meridional wind amplitudes depend on the latitude, with values ranging from 3 to $6 \mathrm{~m} / \mathrm{s}$ and peaking at $\sim 79^{\circ} \mathrm{S}$ (see Fig. 7 ). This latitudinal behaviour will be used to constrain the meridional structure of the disturbance (see Section 5.2). As to the phase of the tidal harmonics, the position of the sine-fit trough is always close to noon, corresponding to the region where the tide accelerates the flow polewards Due to the smaller 

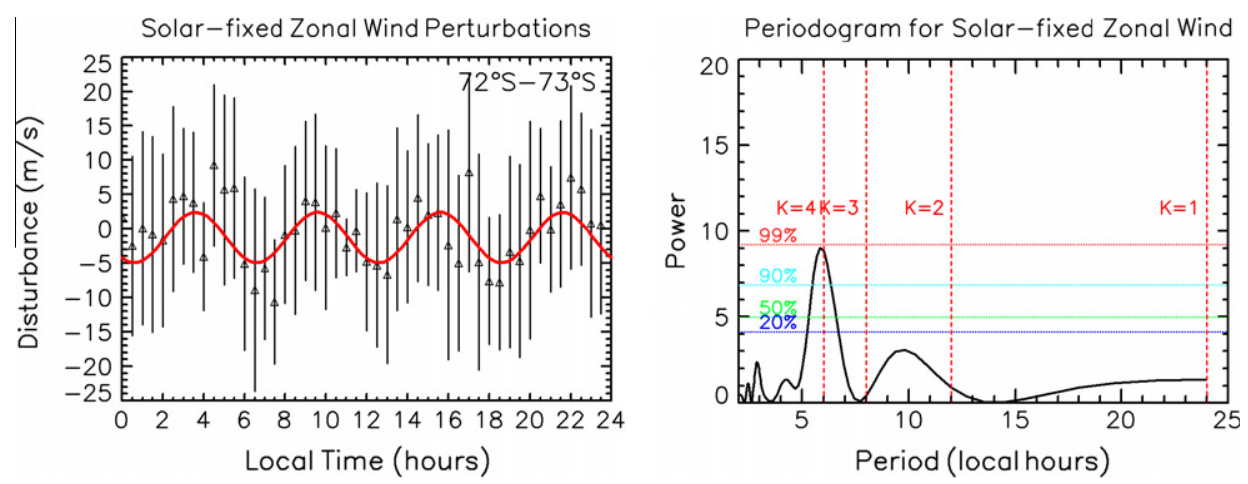

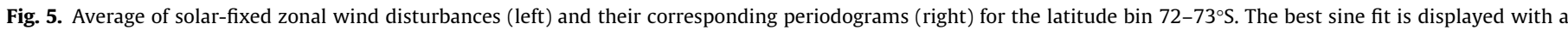

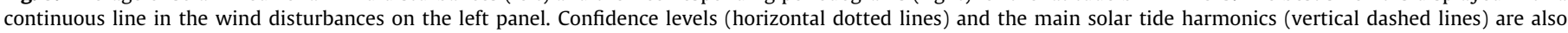
marked in the periodogram on the right.

magnitude of the meridional component in this latitude range (see Fig. 1B), it seems clear that the solar tides drastically affect the direction of the meridional wind, determining whether it is polewards or equatorwards. Thus, the diurnal tides accelerate the atmosphere polewards in the dayside and equatorwards in the nightside (see Fig. 8).

\section{Discussion}

\subsection{Presence of solar tide harmonics in the wind field}

The diurnal tide (wavenumber 1 ) has clearly been detected at southern polar latitudes $\left(70-85^{\circ} \mathrm{S}\right)$ in the meridional component of the wind. A quarter-diurnal tide (wavenumber 4) is apparent at high latitudes in the zonal wind component too, but this requires further confirmation at higher statistical significance. This tidal harmonic was first detected in Venus by Apt et al. (1980) with ground-based observations, and later by Taylor et al. (1980) with Pioneer Venus results. Its presence at latitudes where the diurnal and semidiurnal tides were detected in the temperature field with similar amplitudes (Zasova et al., 2007) could be the result of nonlinear interactions between these diurnal and semidiurnal tides, as occurs in the Earth (Smith and Ortland, 2001; Egbert et al., 2010).

The presence and predominance of the diurnal tide in our analysis of VIRTIS/Venus Express data is consistent with the previous detection of a diurnal tide in the interval $70-80^{\circ} \mathrm{N}$, obtained from longitudinal variations in the atmospheric temperature field retrieved by the OIR instrument during the Pioneer Venus mission (Schofield and Diner, 1983; Schofield and Taylor, 1983). Zasova et al. (2002) discovered in Venera-15 data that for temperature and aerosol concentrations the effect of the solar tide harmonics varied with latitude and altitude, with the diurnal tide starting to be dominant in the cold collar at the cloud tops. Later, this was confirmed from combined thermal data from VIRA, VEGA balloons, Magellan, Venera-15 and Venera-16 (Zasova et al., 2006, 2007). Moreover, Grassi et al. (2010) and Migliorini et al. (2012) have also suggested signatures of a diurnal tide at high latitudes and semidiurnal tide at lower latitudes based on their analysis of VIRTIS data. In contrast to this, radio-occultation data from the VeRa instrument onboard Venus Express (Tellmann et al., 2009) did not detect a wavenumber 1 structure, but a wavenumber 2 (semidiurnal) mode at $\sim 62 \mathrm{~km}$, with a wave amplitude of $15 \mathrm{~K}$ and with minima at the subsolar and antisolar points. However, caution is suggested when interpreting this specific result from VeRa since, due to dataset coverage limitations, the sample used for the sine fit had only 12 bins and the data had a high dispersion. The Pioneer Venus OIR radiometer also obtained a single semidiurnal tide in the tem- peratures inferred from the cloud tops to about $95 \mathrm{~km}$ (Schofield and Taylor, 1983). This result was valid for low latitudes, and the absence of the diurnal tides was explained as the result of poor vertical resolution in the retrieval process; this was due to the halfwidths of the weighting functions for the OIR retrievals being close to or higher than the short vertical wavelengths expected for the diurnal tides (Forbes, 2004).

The atmospheric region studied in our work is restricted to the polar collar, a strong inversion layer apparent between $60-70 \mathrm{~km}$ of altitude and $60-80^{\circ}$ of latitude, which seems to have a solarfixed component (Taylor et al., 1980). This inversion layer was first observed in the northern hemisphere by Pioneer Venus (Taylor et al., 1980; Kliore and Patel, 1982; Newman et al., 1984), and in the southern hemisphere by Venera 15 and 16 (Yakovlev et al., 1991) and, more recently, by Venus Express (Grassi et al., 2008; Tellmann et al., 2009). Nevertheless, the persistent nature of the cold collar is still doubtful as it was not seen in some southern hemisphere profiles from Pioneer Venus (Kliore and Patel, 1982) nor was it detected in the three northern hemisphere occultation profiles retrieved from Magellan (Jenkins et al., 1994). Moreover, zonal and meridional decoupling among different harmonics is an expected tidal feature for regions with isothermal conditions (Volland, 1988), which in turn is a characteristic of the polar collar region in both hemispheres as confirmed by most studies (Taylor et al., 1980; Yakovlev et al., 1991; Jenkins et al., 1994; Tellmann et al., 2009).

\subsection{Spatial structure for the diurnal tide}

Despite the limited latitudinal coverage of the wind disturbances in our study $\left(\sim 15^{\circ}\right)$, a clear variation of the tidal amplitude can be seen (see Fig. 7). Such prominent variations at high latitudes were anticipated by Elson (1983), whose numerical model for Venus showed meridional velocity components to be affected by latitudinal gradients in the geopotential field in the polar region. Unfortunately, due to the limited latitudinal coverage of our dataset it is not possible to fit our results using several Legendre polynomials (Eq. (13)) as it is usually done for the Earth (Lindzen and Chapman, 1969). However, since Limaye (2007) extracted from cloud-tracked winds with Pioneer Venus images a wider meridional coverage (equator and midlatitudes) of the diurnal tide amplitude in the meridional wind, we used his results to infer the meridional structure by means of a single Legendre polynomial fit. The resulting meridional wavenumber allowed the estimation of the vertical wavenumber (see Eq. (12)). From the best fit in terms of Legendre polynomials we obtain a meridional wavenumber 6 for the diurnal tide in our results and a meridional wavenumber 2 for the tidal components determined from Pioneer 

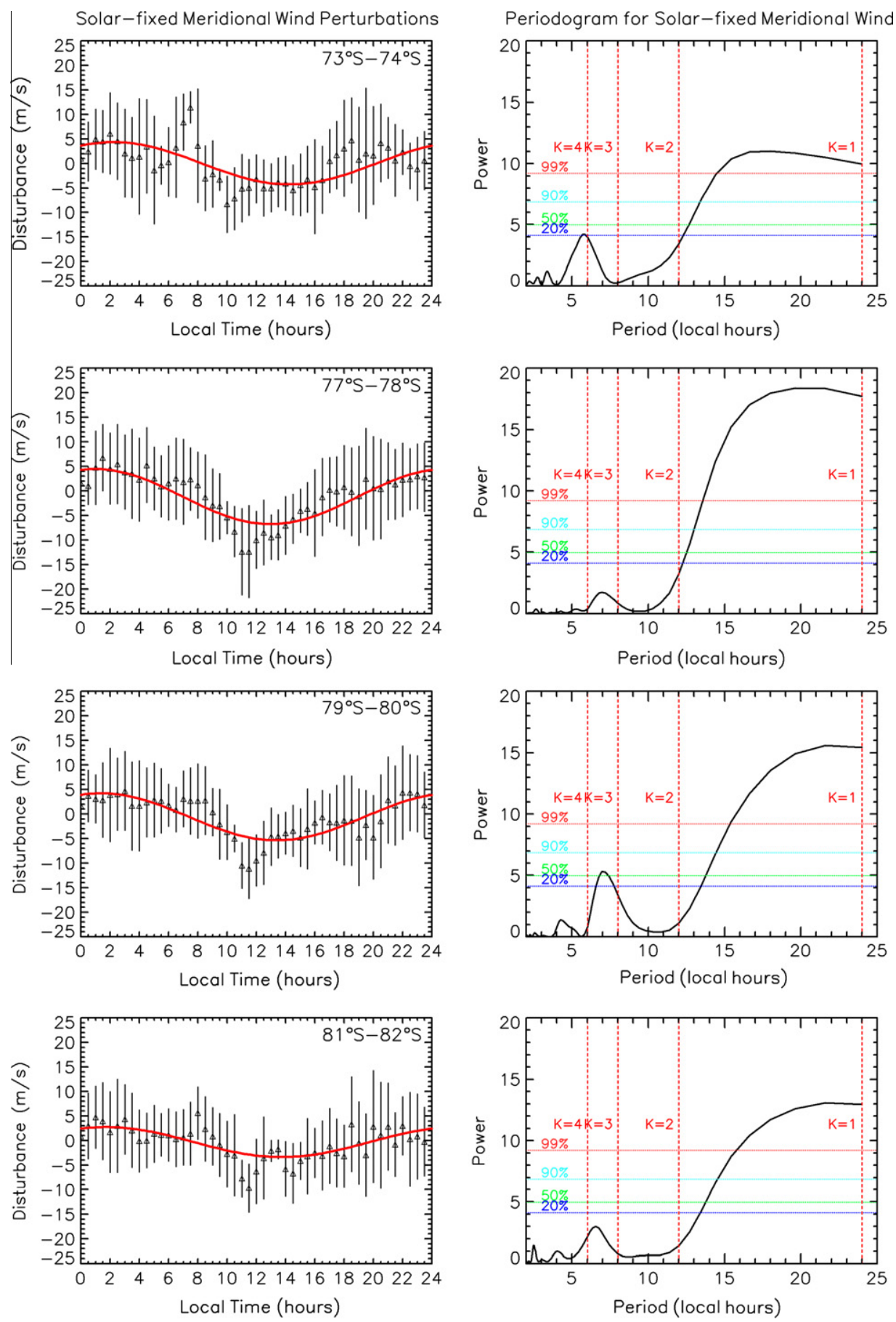

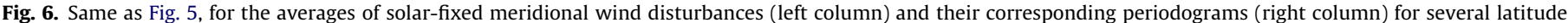
bins.

Venus, though due to its wider coverage only the fit for the Pioneer Venus has been taken into consideration. Future studies should extend the tidal analysis to lower latitudes in order to make a more reliable fit and confirm the meridional structure of the diurnal tide.

The absence at high latitudes of a diurnal tide in the zonal component of the wind could also be related to the global horizontal structure of the diurnal tide. With the source of excitation being the periodic heating by the Sun, an important temperature gradient is expected between the subsolar and antisolar regions, leading to wave fronts approximately tangent to the lines of constant solar zenith angle (SZA). As a result, the corresponding pressure gradients would be perpendicular to the SZA isolines and the solar tide would induce a flow circulation from the solar to the antisolar point, as illustrated in Fig. 9A.

One of the main consequences of this tidal structure is that the diurnal tide acceleration will be mainly zonal for lower latitudes and meridional for higher latitudes, except close to the morning and evening terminator, where the tide forces prograde and retrograde accelerations, respectively, at all latitudes. This tendency is more clearly shown in the two graphs in panel 9B, where the shaded region represents the latitude range of our study. It can be directly concluded that most of the measurements within the solar-fixed series of wind disturbances between $70^{\circ} \mathrm{S}$ and $85^{\circ} \mathrm{S}$ are affected by 

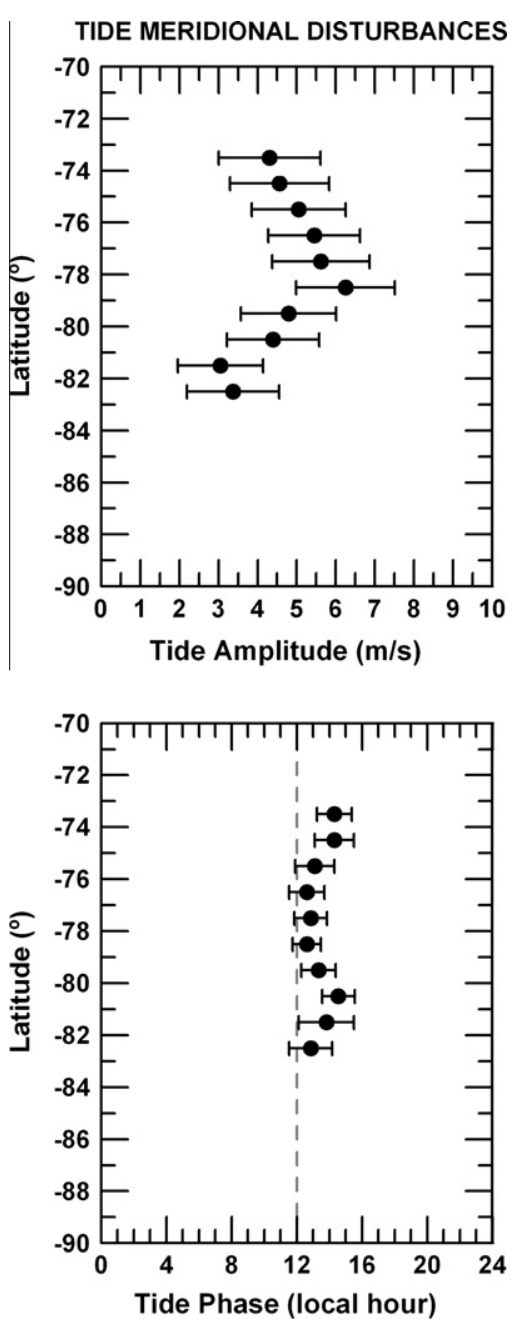

Fig. 7. Latitudinal behaviour for the diurnal tide's amplitude and phase in the meridional wind, in the range $70-85^{\circ} \mathrm{S}$. The top panel displays the tidal amplitude and the bottom one the tidal phase. The phase has been defined as the position of the highest tidal acceleration in the poleward sense. Noon is marked with a grey dashed line.

the diurnal tide in the meridional direction rather than the zonal, which is consistent with our results. A similar horizontal structure was also obtained by Takagi and Matsuda (2005) in their numerical study about the influence of the background zonal flow and the Newtonian cooling on the thermal tides in Venus. Their simulations indicate that it is the zonal flow that mostly affects the resulting horizontal structure of the diurnal tide, and that the diurnal tide mostly affects the horizontal wind in the meridional direction at latitudes 70-90 (see Fig. 6 in Takagi and Matsuda (2005)).

Once we have an estimation for both the zonal and the meridional planetary wavenumber for the diurnal tide (i.e., $\hat{k}_{x}=1$ and $\hat{k}_{y}=2$ ), and assuming the validity of the assumptions leading to Eq. (12) we can also infer the vertical wavelength for the diurnal tide. Thus, the dispersion relation (12) can be modified to obtain, in the diurnal tide case $(j=1)$, the vertical wavelength $\lambda_{z}$ in terms of the planetary zonal and meridional wavenumbers:

$\lambda_{z}=\left|\Omega-\frac{u_{0}}{(a+z) \cdot \cos \phi} \hat{k}_{x}\right| \cdot \frac{2 \pi \cdot(a+z)}{N \cdot \sqrt{\hat{k}_{x}^{2} \cdot \sec ^{2} \phi+\hat{k}_{y}^{2}}}$.

We assume the following values: $\hat{k}_{x}=1, \hat{k}_{y}=2, a+z=$ $6116.5 \mathrm{~km}$ (an altitude of $65 \mathrm{~km}$ ), $\Omega=6.22 \times 10^{-7} \mathrm{~s}^{-1}$ (solar day of 117 terrestrial days) and $N^{2}=260 \times 10^{-6} \mathrm{~s}^{-2}$ (a typical Brunt-Väisälä frequency at about $65 \mathrm{~km}$ for latitudes in the range

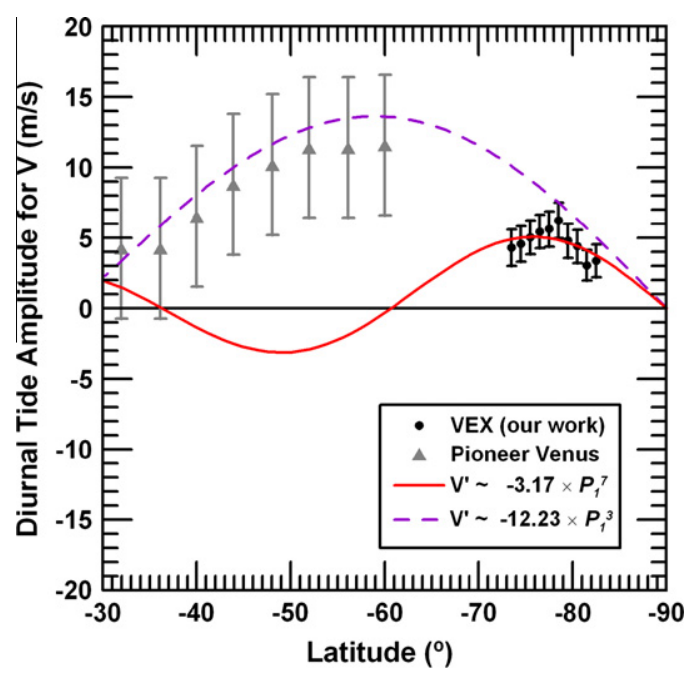

Fig. 8. Meridional structure for the amplitude of the diurnal tide affecting the meridional component of the wind. Our results are plotted with black circles, while the grey triangles correspond to cloud tracking results from UV images with the Pioneer Venus OCPP instrument (Limaye, 2007). Best fits to Legendre polynomials are also shown, with a continuous red line for Venus Express and dashed purple line for Pioneer Venus. A meridional wavenumber 2 is obtained for Pioneer Venus winds, while 6 is the best fit for our results. Numerical values are indicated in the legend.
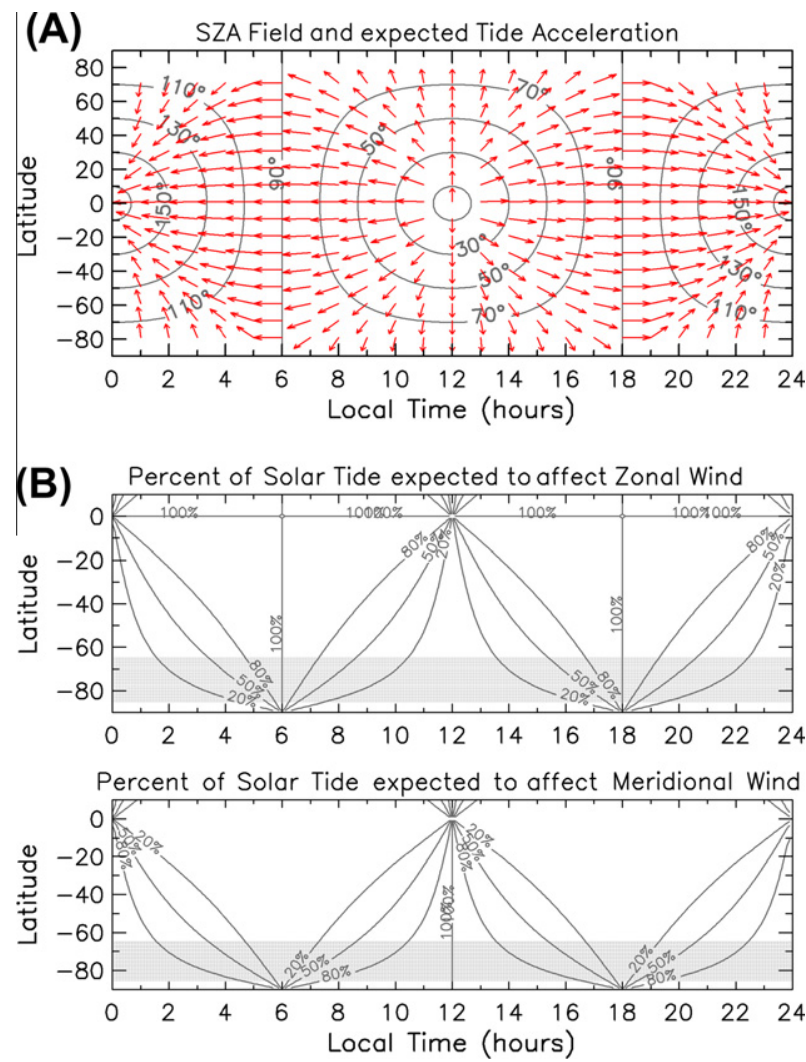

Fig. 9. The latitude vs. solar local time map of the expected solar-to-antisolar flux for the diurnal tide (panel A) and percentages of tidal contribution on the zonal and meridional component of the wind (panel B). The wave fronts are assumed to be tangent to the lines of constant solar zenith angle (line contours) while the red arrows indicate both negative pressure gradients and the sense of the tidal wind acceleration (panel A). The percentage of total tidal contribution affecting each component of the wind is shown in panel B with contour lines and the region of our study shaded in grey. (For interpretation of the references to colour in this figure legend, the reader is referred to the web version of this article.) 
$70-85^{\circ}$ S; see Piccialli, 2010). If we also consider the horizontal shear of the background zonal wind in the region of study, we obtain a value of $\lambda_{z} \approx 8 \pm 2 \mathrm{~km}$. As it happens in the Earth (Volland, 1988), the diurnal tide has a vertical wavelength higher than the scale height, being in this case the cloud scale height that ranges from 1 to $5 \mathrm{~km}$ for the cloud tops at high latitudes (Ignatiev et al., 2009). This estimation of the vertical wavelength is also consistent with previous studies: Fels (1986) showed that vertically propagating diurnal tides should have vertical wavelengths $<10 \mathrm{~km}$, while numerical modeling studies by Pechmann and Ingersoll (1984) showed that the diurnal tide between altitudes of 60 and $90 \mathrm{~km}$ is characterized by an upward wave with a vertical wavelength of about $7 \mathrm{~km}$. Recent results from the VeRa instrument onboard Venus Express also allowed temperature fluctuations with vertical wavelengths of only a few kilometres to be detected that could indicate the presence of the diurnal tide between 40 and $90 \mathrm{~km}$ (Tellmann et al., 2011) but their horizontal structure remains to be determined.

\subsection{Tidal amplitude and phase: implications for the atmospheric circulation}

As mentioned previously, the effect of the solar tides on the cloud-top wind velocities was documented by Limaye (1988), Rossow et al. (1990), and more recently by Limaye (2007). The effect of the solar tides was then characterized using images taken by the OCPP instrument during the Pioneer Venus mission, from the equator up to latitudes of $60^{\circ}$, hence excluding the region covered in our study. Rossow et al. (1990) found both diurnal and semidiurnal tides in the zonal component. Moreover, the amplitudes of the disturbances varied with latitude, with the highest ones $(\sim 10 \mathrm{~m} /$ s) at the equator and amplitudes up to $4 \mathrm{~m} / \mathrm{s}$ at high latitudes $\left(50^{\circ}\right)$. On the other hand, Limaye (2007) found tidal amplitudes of about $15-20 \mathrm{~m} / \mathrm{s}$ at latitudes of $60^{\circ}$, but warned of the high dispersion due to the scarce number of measurements in this region. In the case of Venus Express, winds obtained with cloud tracking in ultraviolet images from VIRTIS-M (Sánchez-Lavega et al., 2008; Hueso et al., 2012) and VMC (Moissl et al., 2009) displayed a clear acceleration of the zonal wind at the $12 \mathrm{~h}$ meridian. This effect was interpreted to be associated with the solar tides but in the case of VIRTIS-M no attempt was made to characterize it beyond the estimation of local-time change $(\sim 2.5 \mathrm{~m} / \mathrm{s}$ per local hour from morning to evening close to $60^{\circ} \mathrm{S}$ ). Moissl et al. (2009) characterized with VMC images the region between the equator and $60^{\circ} \mathrm{S}$ and a local time region ranging from $9 \mathrm{~h}$ to $16 \mathrm{~h}$, and found indications of the diurnal and semidiurnal effect on the zonal wind, with amplitudes of about $15 \mathrm{~m} / \mathrm{s}$, consistent with Pioneer Venus results. None of the previous studies with Venus Express, however, analyzes the effect of solar tides on the meridional component of the wind.

According to our results, this strong acceleration detected for the zonal wind at the polar collar latitudes by Sánchez-Lavega et al. (2008) and Moissl et al. (2009) cannot be explained in terms of the diurnal tide as it does not affect the zonal component at those latitudes. An estimation of the tidal phase and amplitude would be required for the winds obtained with ultraviolet images to confirm whether these accelerations are spatially periodic and, thus, an effect of the diurnal tide. The effect of measuring at different dates cannot be discarded as Rossow et al. (1990) found that while the semidiurnal amplitude was constant, the diurnal one seemed subject to a temporal variability. Since the solar tides are triggered by solar heat deposition, any change in the cloud structure (for example due to dynamical reasons) could also change the heating rate and, thus, the tidal amplitude.

As stated before, mean wave amplitudes of $\sim 4.7 \mathrm{~m} / \mathrm{s}$ were found for the diurnal tide in the meridional direction. Compared with the small values for the mean meridional wind in the polar collar (see Fig. 1A), these amplitudes clearly imply a crucial role for the diurnal tides, which clearly determine the flow direction (polewards or equatorwards). Moreover, the spatially periodic behaviour of the tidal wavenumber 1 mode explains the null values found for the zonally averaged meridional velocities. Since the sine trough is close to noon, another important conclusion is that at high latitudes the diurnal tide accelerates the wind polewards in the dayside and equatorwards in the nightside, suggesting the flow to be dominated by a solar-antisolar circulation. Poleward of $85^{\circ} \mathrm{S}$ the solar tides are not apparent, this is probably due to the complex contribution of the southern polar vortex to the atmospheric circulation there (Luz et al., 2011).

Meridional winds inferred with UV images from previous missions to Venus (Rossow et al., 1990; Belton et al., 1991; Peralta et al., 2007) and also from VIRTIS-M onboard Venus Express (Sánchez-Lavega et al., 2008, Fig. 2b) exhibit a poleward circulation extending from the equator to about $70^{\circ} \mathrm{S}$. This meridional dayside circulation at the top of the clouds has been classically interpreted as the upper branch of a Hadley-cell circulation extending from the equator to latitudes between $50^{\circ}$ and $60^{\circ}$ (Schubert, 1983; Limaye, 2007; Peralta et al., 2007), and this interpretation are also supported by recent results with Venus GCMs (Lee et al., 2007; Hollingsworth et al., 2007). Nevertheless, the nightside sense of the meridional circulation at the cloud tops still needs to be confirmed for lower latitudes, and in situ measurements by Pioneer Venus Night and North probes are contradictory (Schubert, 1983). Our new findings introduce a dayside poleward circulation forced by the diurnal tide poleward of $70^{\circ} \mathrm{S}$ that is compatible with the existence of an axisymmetric Hadley circulation in Venus, although the reported tidal-forced equatorward circulation at high latitudes of the nightside coupled with a Hadley poleward circulation would, then, imply an interesting case of converging flows not predicted by the models. On the other hand, the finding of equatorward winds at the lower latitudes in the nightside would confirm the existence of a superimposed solar-to-antisolar circulation in the cloud region due to the diurnal tide, supported by computer simulations by Newman and Leovy (1992) or Takagi and Matsuda (2005).

\subsection{Associated thermal effects for the diurnal tide}

In Section 2.2 we derived for the diurnal tide an expression relating the tidal amplitudes of the temperature disturbances with the background mean zonal flow and the tidal wind disturbances (Eq. (10)). Since the diurnal tide has only been detected in the meridional component of the wind, the corresponding thermal tide amplitudes were calculated and their latitudinal behaviour compared with the tide amplitudes obtained with Pioneer Venus OIR radio-occultation data (channel 5 , corresponding to a vertical level of about $65 \mathrm{~km}$; Taylor et al., 1980), the Venera-15 measurements corresponding to a vertical level of $60 \mathrm{~km}$ (Zasova et al., 2007) and with results from the numerical model by Pechmann and Ingersoll (1984) which simulated the same level as PV-OIR Chanel-5 (see Fig. 10).

The best agreement is found between our results and Pechmann's model. Discrepancies with Venera-15 are small, and the largest discrepancy is with Pioneer Venus poleward of $80^{\circ}$. Nevertheless, Forbes (2004) suggests caution when using the OIR temperature retrievals to infer the diurnal tide amplitudes, as the retrieved temperatures are a smoothed version of the true profile: the OIR weighting functions are broad and this probably conflicts with the small wavelength expected for the diurnal tides. Grassi et al. (2010) also suggested that tidal effects may be present in the solar-fixed temperatures retrieved with VIRTIS at $100 \mathrm{mb}$ in the nightside ( $18 \mathrm{~h}$ to $6 \mathrm{~h} \mathrm{LT}$ ), finding at $75^{\circ} \mathrm{S}$ and $85^{\circ} \mathrm{S}$ temperature 


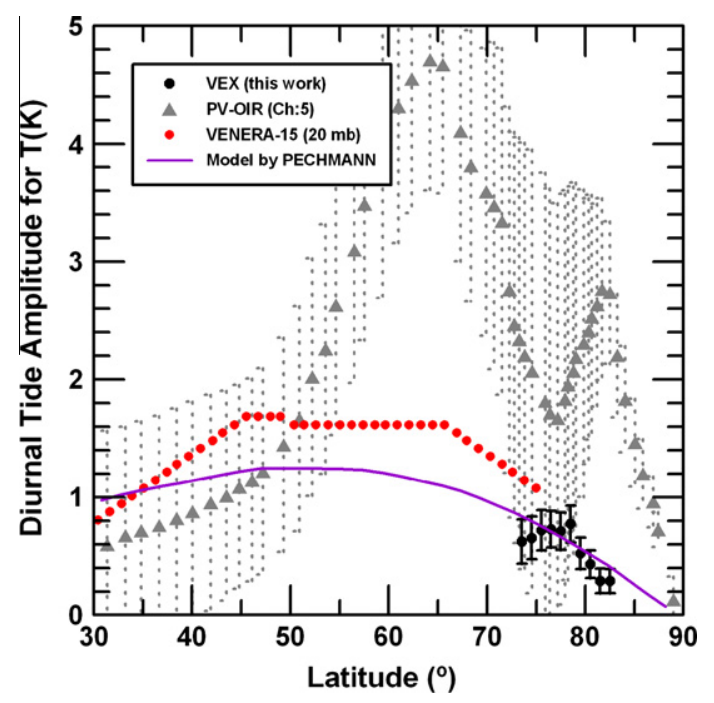

Fig. 10. Meridional structure of the diurnal tide inferred for the temperature. Our results with Venus Express (black circles) are plotted together with Pioneer Venus OIR radio-occultation ( $\sim 65 \mathrm{~km})$ measurements (grey triangles, Taylor et al., 1980) and with Venera-15 measurements ( $60 \mathrm{~km}$, red circles, Zasova et al., 2007). The purple line displays the Pechmann and Ingersoll (1984) tidal model results $(\sim 65 \mathrm{~km})$. (For interpretation of the references to colour in this figure legend, the reader is referred to the web version of this article.)

deviations lower than $4 \mathrm{~K}$ but with standard deviations higher than $9 \mathrm{~K}$. The discrepancies between our inferences and PV-OIR channel 5 for the thermal tide amplitude range from 1 to $2 \mathrm{~K}$, and the poleward decrease in the tidal temperature amplitude in Venera measurements and modelling by Pechmann and Ingersoll (1984) is also apparent in our results. This argues in favour of the validity of Eq. (10) relating tidal disturbances for temperatures and wind velocity.

\section{Conclusions}

As indicated by measurements of temperature fields by previous missions, the diurnal tide (wavenumber 1 ) is the dominant solar harmonic in the polar collar region, and is only detected in the meridional wind. This is compatible with a spatial distribution of wave fronts tangent to the lines of constant solar zenith angle, a horizontal structure already predicted by models (Takagi and Matsuda, 2005) and to be confirmed with measurements covering lower latitudes. A weaker quarter-diurnal (wavenumber 4) mode is also apparent in the zonal component of the wind at a narrow range of latitude and average amplitudes of $\sim 2.2 \mathrm{~m} / \mathrm{s}$ but requires confirmation. The diurnal tide amplitude has a mean value of $4.7 \mathrm{~m} / \mathrm{s}$, which implies that in the absence of other effects it determines the sense of the meridional flow. The diurnal and quarter-diurnal tides affecting the meridional and zonal winds respectively, seem to be decoupled, as it is expected for tidal harmonics under isothermal conditions (a well known property of the polar collar region).

Due to the restricted meridional coverage of our data we estimated the meridional wave structure of the diurnal tide using the Pioneer Venus wind measurements, obtaining a meridional wavenumber 2 . The vertical structure was subsequently estimated using the dispersion equation derived from the Taylor-Goldstein equation for the case of large scale gravity waves (Nappo, 2002). This yielded a vertical wavelength of about $8 \mathrm{~km}$, in agreement with theoretical and numerical model results. The phase of the diurnal tide does not vary with the latitude, implying poleward fluid motion in the dayside and equatorward in the nightside. This is consistent with a solar-to-antisolar circulation induced by the solar tides at the cloud tops of the polar region.

Finally, an expression relating the tidal amplitude of disturbances in temperature and wind velocity has been derived from the primitive equations, which allowed the calculation of the corresponding tide effects on the atmospheric temperature. Our expression proves to be a good approximation when compared with results from previous missions and numerical models. Specifically, excellent agreement was found with the model by Pechmann and Ingersoll (1984), while discrepancies lower than $2 \mathrm{~K}$ occur with PV-OIR measurements.

Our characterization of the influence of atmospheric solar tides on the wind field on Venus implies a forward step to find out the role played by these global-scale waves in the general circulation of Venus. It also provides important constraints on Venus general circulation models, and we hope that upcoming measurements from Venus Express and ground-based observations will serve to both confirm our results and extend the characterization of the solar tides to lower latitudes of the planet.

\section{Acknowledgments}

J. Peralta acknowledges support from the Portuguese Foundation for Science and Technology (FCT, Grant reference: SFRH/BPD/ 63036/2009). J.P., D.L. and D.B. also acknowledge FCT funding through Projects POCI/CTE-AST/110702/2009 and PEst-OE/FIS/ UI2751/2011. RH and ASL are supported by the Spanish MICIIN Project AYA2009-10701 with FEDER and Grupos Gobierno Vasco IT-464-07. Venus Express is a mission of the European Space Agency. VIRTIS was supported by CNES (Centre National d'Etudes Spatiales) and ASI (Agenzia Spaziale Italiana). We are grateful to all members of the ESA Venus Express project and of the VIRTIS technical team, in addition to the invaluable revision by Dr. Masahiro Takagi and another anonymous reviewer.

\section{Appendix A. Classical tidal theory and its applicability to the Venus case}

The tidal structure is classically described in terms of solutions to the Laplace Tidal Equation (hereafter LTE), that can be derived from the primitive equations of motion assuming several conditions for the atmosphere (Laplace et al., 1832). We will not display a detailed derivation of this equation but a brief summary, and we refer the reader to the abundant bibliography for the intermediate steps to its derivation (Lindzen and Chapman, 1969; Volland, 1988). The following assumptions are usually accepted as valid for the terrestrial planets: (a) planet's ellipticity, surface topography, and main dissipative processes can be ignored; (b) the atmosphere is in hydrostatic equilibrium, the shallow atmosphere equilibrium can be applied and it can be described with the Navier-Stokes equations; (c) the atmosphere behaves as a perfect gas of constant composition and in thermodynamic equilibrium; and (d) tidal fields are perturbations which can be linearized about a basic state that behaves as steady with a mean zonal flow equal to zero.

The perturbation fields are, then, assumed to have sinusoidal variations in longitude and time, in the form:

$F^{m, \sigma}(\lambda, \theta, z, t)=f^{m, \sigma}(\theta, z) \cdot e^{i(m \lambda-\alpha)}$,

where $\sigma=2 \pi \cdot j(1 \text { solar day })^{-1}$ is the angular frequency in units of the solar day, $j=1,2, \ldots$ are the possible solar harmonics, $m$ is in this case the zonal wavenumber, $\lambda$ is the longitude, $\theta$ is the colatitude and $z$ is the altitude. Under the mentioned assumptions, some of the physical unknowns in the primitive equations (among them, 
the atmospheric temperature) allow separating the variables $\theta$ and $z$, and are expressed with the form:

$f^{m, \sigma}(\theta, z)=\sum_{n} C_{n}^{m, \sigma}(z) \cdot \Theta_{n}^{m, \sigma}(\theta)$,

where the coefficients $C_{n}^{m, \sigma}(z)$ are related to the heating function (Sánchez-Lavega, 2011) and $\Theta_{n}^{m, \sigma}$ are Hough functions, a special case of series of Legendre polynomials with the form:

$\Theta_{n}^{m, \sigma}=\sum_{s=1}^{\infty} c_{s, n}^{m}(\sigma) \cdot P_{s}^{m}(\theta)$.

Manipulating the primitive equations with this separation of variables one can arrive at the following equations:

$\mathfrak{J}\left(\Theta_{n}^{m, \sigma}\right)=-\varepsilon_{n} \cdot \Theta_{n}^{m, \sigma}$,

$\frac{\partial^{2} W_{n}^{m, \sigma}}{\partial P^{2}}+\frac{S_{P}}{g \cdot h_{n}}=\frac{-R}{g \cdot h_{n} \cdot c_{P}} \cdot\left(\frac{J_{n}^{m, \sigma}}{P}\right)$

where $\mathfrak{I}$ in Eq. (A4) is an operator function of the latitude, the zonal wavenumber $m$ and the normalized frequency $v=\sigma / 2 \Omega$ (Lindzen and Chapman, 1969). The non-dimensional quantity $\varepsilon_{n}=\frac{4 \Omega^{2} a^{2}}{h_{n} g}$ in (A3) is called the "planet constant", $h_{n}$ is a separation constant called "equivalent depth", $a$ is the planetary radius, $g$ is the gravity acceleration, $\Omega$ is the frequency for the sidereal day, $S_{P}$ is the static stability parameter, $c_{P}$ is the heat capacity at constant pressure, $R$ is the gas constant, $P$ is the pressure, $\omega^{m, \sigma}=\sum_{n} W_{n}^{m, \sigma}(z) \cdot \Theta_{n}^{m, \sigma}(\theta)$ is the isobaric vertical velocity, and $Q^{m, \sigma}=\sum_{n} J_{n}^{m, \sigma}(z) \cdot \Theta_{n}^{m, \sigma}(\theta)$ is the heating function. Eq. (A4) is known as the "meridional structure equation" or LTE, as previously mentioned. Eq. (A5) is the "vertical structure equation".

The LTE is a second order differential equation whose solutions are the eigenvalues $\varepsilon_{n}$ and the eigenfunctions $\Theta_{n}^{m, \sigma}$. The LTE is a universal equation that can be applied to any planet whose atmosphere is thin compared with its radius, as it contains only two non-dimensional parameters. This implies that for given $m$ and $v$, the eigenvalues and eigenfunctions of the LTE will be the same for all planets, independently of the absolute rotation rate, mass, radius or thermodynamics of the atmosphere (Lindzen, 1970). And it can be demonstrated (Volland, 1988) that the eigenfunctions solution of the LTE are Hough functions.

The Hough functions $\left(\Theta_{n}\right)$ and their corresponding equivalent depths $\left(h_{n}\right)$ can be found conveniently tabulated as solutions of Eq. (A4) and are appropriate to describe tidal fields such as the vertical velocity, pressure, density and temperature, while for the zonal and meridional velocities the formula is different and more complex (Lindzen and Chapman, 1969).

Lindzen (1970) noted several considerations for the applicability of the Hough functions expansion to the Venus case. On the one hand, the non-dimensional planet constant $\varepsilon_{n}$ in the expression $\varepsilon_{n}=\frac{4 \Omega^{2} a^{2}}{h_{n} g}$ is, in fact, an eigenvalue applicable to any planet. Therefore, combining it with the venusian values for $\Omega, g$ and $a$ we can infer the Venus equivalent depth $h_{n}$. On the other hand, while both the solar and sidereal days have the approximate value of $24 \mathrm{~h}$ for the Earth, in the case of Venus these are 117 and 247 days respectively; so, as one is nearly twice the other, Lindzen (1970) showed that the Hough functions and their corresponding planetary constants for the diurnal tide on Venus would then match the ones for the semidiurnal tides in the Earth.

Once the expansion of Hough functions describing the meridional distribution of an atmospheric parameter has been found, we can also introduce in Eq. (A5) the equivalent depths to obtain information on the vertical structure, type and vertical wavelength. The vertical structure Eq. (A5) can be written in a simpler form using the following change of variables: $z \equiv \ln \left(\frac{P_{0}}{P}\right)=-\ln \left(\frac{P}{P_{0}}\right)$,

$W_{n}^{m, \sigma} \equiv-\gamma \cdot P \cdot e^{Z / 2} \cdot Y_{n}^{m, \sigma}=-\gamma \cdot P_{0} \cdot e^{-Z / 2} \cdot Y_{n}^{m, \sigma}$,

where $\gamma=c_{p} / c_{V}$ and $Z$ is a non-dimensional parameter. In case of an isothermal atmosphere, $d z=\frac{R T}{g} D Z$ and $Z$ can be considered as a non-dimensional altitude. Then, rewriting (A4) we have:

$\frac{\partial^{2} Y_{n}^{m, \sigma}}{\partial Z^{2}}-\frac{1}{4} \cdot\left[1-\frac{4}{h_{n}}\left(\kappa \cdot H+\frac{\partial H}{\partial Z}\right)\right] \cdot Y_{n}^{m, \sigma}=\frac{\kappa \cdot J_{n}^{m, \sigma}}{\gamma \cdot g \cdot h_{n}} \cdot e^{-Z / 2}$,

where $\kappa=R / c_{p}$ and $H=R T / g$. If we now denote,

$\Gamma^{2} \equiv \frac{1}{4} \cdot\left[1-\frac{4}{h_{n}}\left(\kappa \cdot H+\frac{\partial H}{\partial Z}\right)\right]$

then, for an isothermal atmosphere $\Gamma^{2}=$ constant, we will have the following solutions depending on its value:

$Y_{n}^{m, \sigma} \sim A \cdot e^{\Gamma \cdot Z}+B \cdot e^{-\Gamma \cdot Z}$ for $\Gamma^{2}>0$,

$Y_{n}^{m, \sigma} \sim A \cdot e^{i \cdot \Gamma \cdot Z}+B \cdot e^{-i \cdot \Gamma \cdot Z} \quad$ for $\Gamma^{2}>0$.

For $\Gamma^{2}>0$, the exponential solution indicates the exponential decay of a disturbance away from the excitation $(A=0$ as one of the border conditions must be $\left.Y_{n}^{m, \sigma}(0)=0\right)$. And for $\Gamma^{2}>0$, the solution is a wave with vertical wavelength $2 \pi /|\Gamma|$, and an upward propagation away from the region of excitation.

\section{References}

Apt, J., Brown, R.A., Goody, R.M., 1980. The character of the thermal emission from Venus. J. Geophys. Res. 85, 7934-7940.

Baker, N.L., Leovy, C.B., 1987. Zonal winds near Venus' cloud top level - A model study of the interaction between the zonal mean circulation and the semidiurnal tide. Icarus 69, 202-220.

Belton, M.J.S. et al., 1991. Images from Galileo of the Venus cloud deck. Science 253, 1531-1536.

Del Genio, A.D., Rossow, W.B., 1990. Planetary-scale waves and the cyclic nature of cloud top dynamics on Venus. J. Atmos. Sci. 47, 293-318.

Drossart, P. et al., 2007. Scientific goals for the observation of Venus by VIRTIS on ESA/Venus Express mission. Planet. Space Sci. 55, 1653-1672.

Egbert, G.D., Erofeeva, S.Y., Ray, R.D., 2010. Assimilation of altimetry data for nonlinear shallow-water tides: Quarter-diurnal tides of the Northwest European Shelf. Cont. Shelf Res. 30, 668-679.

Elson, L.S., 1983. Solar related waves in the venusian atmosphere from the cloud tops to $100 \mathrm{~km}$. J. Atmos. Sci. 40, 1535-1551.

Fels, S.B., 1986. An approximate analytical method for calculating tides in the atmosphere of Venus. J. Atmos. Sci. 43, 2757-2772.

Fels, S.B., Lindzen, R.S., 1974. The interaction of thermally excited gravity waves with mean flows. Geophys. Astrophys. Fluid Dyn. 5, 211-212.

Forbes, J.M., 2004. Tides in the middle and upper atmospheres of Mars and Venus. Adv. Space Res. 33, 125-131.

Frescura, F.A.M., Engelbrecht C.A., Frank B.S., 2007. Significance Tests for Periodogram Peaks. arXiv:0706.2225.

Gierasch, P.J. et al., 1997. The general circulation of the Venus atmosphere: An assessment. In: Venus II: Geology, Geophysics, Atmosphere, and Solar Wind Environment. University of Arizona Press, p. 459.

Grassi, D. et al., 2008. Retrieval of air temperature profiles in the venusian mesosphere from VIRTIS-M data: Description and validation of algorithms. J. Geophys. Res. 113, Е00B09.

Grassi, D. et al., 2010. Average thermal structure of venusian night-time mesosphere as observed by VIRTIS-Venus Express. J. Geophys. Res. 115, E09007.

Hollingsworth, J.L., Young, R.E., Schubert, G., Covey, C., Grossman, A.S., 2007. A simple-physics global circulation model for Venus: Sensitivity assessments of atmospheric superrotation. Geophys. Res. Lett. 34, L05202.

Holton, J.R., 2004. An Introduction to Dynamic Meteorology, fourth ed. Elsevier, New York.

Holton, J.R., Pyle, J., Curry, J.A., 2002. Encyclopedia of Atmospheric Sciences. Elsevier Science. ISBN 100122270908.

Hou, A.Y., Fels, S.B., Goody, R.M., 1990. Zonal superrotation above Venus' cloud base induced by the semidiurnal tide and the mean meridional circulation. J. Atmos. Sci. 47, 1894-1901.

Houghton, J., 2002. The Physics of Atmospheres. Cambridge University Press, p. 336. ISBN 0521804566

Hueso, R., Peralta, J., Sánchez-Lavega, A., 2012. Assessing the long-term variability of Venus winds at cloud level from VIRTIS-Venus Express. Icarus 217, 585-598. 
Ignatiev, N.I. et al., 2009. Altimetry of the Venus cloud tops from the Venus Express observations. J. Geophys. Res. 114, E00B43.

Ingersoll, A.P., Orton, G.S., 1974. Lateral inhomogeneities in the Venus atmosphere: Analysis of thermal infrared maps. Icarus 21, 121-128.

Jenkins, J.M., Steffes, P.G., Hinson, D.P., Twicken, J.D., Tyler, G.L., 1994. Radio occultation studies of the Venus atmosphere with the Magellan spacecraft. 2: Results from the October 1991 experiments. Icarus 110, 79-94.

Kliore, A.J., Patel, I.R., 1982. Thermal structure of the atmosphere of Venus from Pioneer Venus radio occultations. Icarus 52, 320-334.

Laplace, P.S., 1832. Méchanique Céleste, 4 vols, (Translated by N. Bowditch), Boston. The Relevant Section is Part I, Book IV, Section 3, p. 543.

Lebonnois, S., Hourdin, F., Eymet, V., Crespin, A., Fournier, R., Forget, F., 2010 Superrotation of Venus' atmosphere analyzed with a full general circulation model. J. Geophys. Res. 115, E06006.

Lee, C., Lewis, S.R., Read, P.L., 2007. Superrotation in a Venus general circulation model. J. Geophys. Res. 112, E04S11.

Limaye, S.S., 1988. Venus: Cloud level circulation during 1982 as determined from Pioneer cloud photopolarimeter images. II - Solar longitude dependent circulation. Icarus 73, 212-226.

Limaye, S.S., 2007. Venus atmospheric circulation - Known and unknown. J. Geophys. Res. 112, E04S09.

Lindzen, R.S., 1966. On the theory of the diurnal tide. Mont. Weather Rev. 94, 295301.

Lindzen, R.S., 1970. The application and applicability of terrestrial atmospheric tidal theory to Venus and Mars. J. Atmos. Sci. 27, 536-549.

Lindzen, R.S., Chapman, S., 1969. Atmospheric tides. Space Sci. Rev. 10, 3-188.

Liu, X., Xu, J., Liu, H.-L., Ma, R., 2008. Nonlinear interactions between gravity waves with different wavelengths and diurnal tide. J. Geophys. Res. 113, D08112.

Luz, D., Berry, D.L., Roos-Serote, M., 2008. An automated method for tracking clouds in planetary atmospheres. New Astron. 13, 224-232.

Luz, D. et al., 2011. Venus's southern polar vortex reveals precessing circulation. Science 332, 577-580.

Migliorini, A., Grassi, D., Montabone, L., Lebonnois, S., Drossart, P., Piccioni, G., 2012. Investigation of air temperature on the nightside of Venus derived from VIRTIS$\mathrm{H}$ on board Venus-Express. Icarus 217, 640-647.

Moissl, R. et al., 2009. Venus cloud top winds from tracking UV features in Venus Monitoring Camera images. J. Geophys. Res. 114, E00B31.

Moroz, V.I., Ekonomov, A.P., Moshkin, B.E., Revercomb, H.E., Sromovsky, L.A., Schofield, J.T., 1985. Solar and thermal radiation in the Venus atmosphere. Adv. Space Res. 5, 197-232.

Nappo, C.J., 2002. An Introduction to Atmospheric Gravity Waves, vol. 85. Academic Press (International Geophysics Series). ISBN 0125140827.

Newman, M., Leovy, C., 1992. Maintenance of strong rotational winds in Venus' middle atmosphere by thermal tides. Science 257, 647-650.

Newman, M., Schubert, G., Kliore, A.J., Patel, I.R., 1984. Zonal winds in the middle atmosphere of Venus from Pioneer Venus radio occultation data. J. Atmos. Sci. 41, 1901-1913.

Pechmann, J.B., Ingersoll, A.P., 1984. Thermal tides in the atmosphere of Venus - Comparison of model results with observations. J. Atmos. Sci. 41, 3290-3313.

Peralta, J., Hueso, R., Sánchez-Lavega, A., 2007. A reanalysis of Venus winds at two cloud levels from Galileo SSI images. Icarus 190, 469-477.

Piccialli, A., 2010. Cyclostrophic Wind in the Mesosphere of Venus from Venus Express Observations. Ph.D Thesis, Technische Universität Braunschweig. 119pp.

Pollack, J.B. et al., 1993. Near-infrared light from Venus' nightside - A spectroscopic analysis. Icarus 103, 1-42.

Press, W.H., Rybicki, G.B., 1989. Fast algorithm for spectral analysis of unevenly sampled data. Astrophys. J. Part 1 338, 277-280.

Press, W.H., Teukolsky, S.A., Vetterling, W.T., Flannery, B.P., 1992. Numerical Recipes in Fortran 77: The Art of Scientific Computing. Cambridge University Press.

Rossow, W.B., del Genio, A.D., Eichler, T., 1990. Cloud-tracked winds from Pioneer Venus OCPP images. J. Atmos. Sci. 47, 2053-2084.

Sánchez-Lavega, A., 2011. An Introduction to Planetary Atmospheres. Taylor \& Francis, ISBN 142006732X.
Sánchez-Lavega, A. et al., 2008. Variable winds on Venus mapped in three dimensions. Geophys. Res. Lett. 35, L13204.

Scargle, J.D., 1982. Studies in astronomical time series analysis - II: Statistical aspects of spectral analysis on unevenly spaced data. Astrophys. J. 263, 835853.

Schofield, J.T., Diner, D.J., 1983. Rotation of Venus's polar dipole. Nature 305, 116 119.

Schofield, J.T., Taylor, F.W., 1983. Measurements of the mean, solar-fixed temperature and cloud structure of the middle atmosphere of Venus. $\mathrm{R}$. Meteorol. Soc. Q. J. 109, 57-80.

Schubert, G., 1983. General circulation and the dynamical state of the Venus atmosphere. In: "Venus" (A83-37401 17-91). University of Arizona Press, Tucson, AZ, pp. 681-765.

Schubert, G., Whitehead, J.A., 1969. Moving flame experiment with liquid mercury: Possible implications for the Venus atmosphere. Science 163, 71-72.

Seiff, A., Schofield, J.T., Kliore, A.J., Taylor, F.W., Limaye, S.S., 1986. Models of the structure of the atmosphere of Venus from the surface to 100 kilometers altitude. Adv. Space Res. 5, 3-58.

Shen, M., Zhang, C.Z., 1990. A numerical solution for thermal tides in the atmosphere of Venus. Icarus 85, 129-144.

Smith, A.K., Ortland, D.A., 2001. Modeling and analysis of the structure and generation of the Terdiurnal Tide. J. Atmos. Sci. 58, 3116-3134.

Svedhem et al., 2007. Venus Express-The first European mission to Venus. Planet. Space Sci. 55, 1636-1652.

Takagi, M., Matsuda, Y., 2005. Sensitivity of thermal tides in the Venus atmosphere to basic zonal flow and newtonian cooling. Geophys. Res. Lett. 32, L02203.

Takagi, M., Matsuda, Y., 2007. Effects of thermal tides on the Venus atmospheric superrotation. J. Geophys. Res. 112, D09112.

Taylor, F.W. et al., 1980. Structure and meteorology of the middle atmosphere of Venus Infrared remote sensing from the Pioneer orbiter. J. Geophys. Res. 85, 7963-8006.

Tellmann, S., Pätzold, M., Häusler, B., Bird, M.K., Tyler, G.L., 2009. Structure of the Venus neutral atmosphere as observed by the Radio Science experiment VeRa on Venus Express. J. Geophys. Res. 114, E00B36.

Tellmann, S., Häusler, B., Pätzold, M., Bird, M.K., Tyler, G.L., 2011. Small-scale temperature fluctuations in the Venus atmosphere as seen by the VeRa Experiment on Venus Express. EPSC 6, Abstract EPSC-DPS2011-1087.

Toigo, A., Gierasch, P.J., Smith, M.D., 1994. High resolution cloud feature tracking on Venus by Galileo. Icarus 109, 318-336.

Tsang, C.C.C., Irwin, P.G.J., Taylor, F.W., Wilson, C.F., 2008. A correlated-k model of radiative transfer in the near-infrared windows of Venus. J. Quant. Spectrosc. Radiat. Trans. 109, 1118-1135.

Volland, H., 1988. Atmospheric Tidal and Planetary Waves. Kluwer Academic Publishers.

$\mathrm{Xu}$, J. et al., 2009a. Seasonal and quasi-biennial variations in the migrating diurnal tide observed by Thermosphere, Ionosphere, Mesosphere, Energetics and Dynamics (TIMED). J. Geophys. Res. 114, D13107.

$\mathrm{Xu}$, J. et al., 2009b. Estimation of the equivalent Rayleigh friction in mesosphere/ lower thermosphere region from the migrating diurnal tides observed by TIMED. J. Geophys. Res. 114, D23103.

Yakovlev, O.I., Matiugov, S.S., Gubenko, V.N., 1991. Venera-15 and -16 middle atmosphere profiles from radio occultations: Polar and near-polar atmosphere of Venus. Icarus 94, 493-510.

Yamamoto, M., Takahashi, M., 2006. Superrotation maintained by meridional circulation and waves in a Venus-like AGCM. J. Atmos. Sci. 63, 3296-3314

Zasova, L., Khatountsev, I.V., Ignatiev, N.I., Moroz, V.I., 2002. Local Time Variations of the middle atmosphere of Venus: Solar related structures. Adv. Space Res. 29 243-248.

Zasova, L.V., Moroz, V.I., Linkin, V.M., Khatuntsev, I.V., Maiorov, B.S., 2006. Structure of the venusian atmosphere from surface up to $100 \mathrm{~km}$. Cosmic Res. 44, 364383.

Zasova, L.V., Ignatiev, N., Khatuntsev, I., Linkin, V., 2007. Structure of the Venus atmosphere. Planet. Space Sci. 55, 1712-1728. 